\title{
IMMAGINI DELLA CINA NEL PENSIERO POLITICO ITALIANO IN ETÀ MODERNA
}

\author{
PAOLO COSTANTINO PISSAVINO (*)
}

Nota presentata dal m.e. Salvatore Veca

(Adunanza del 6 aprile 2017)

\begin{abstract}
SunTO. - Lo studio che qui si presenta è volto ad analizzare le relazioni che intercorrono tra le immagini della Cina elaborate negli scritti di viaggiatori e di padri gesuiti e i grandi temi - ragion di stato, utopia, costituzione mista - che scandirono il pensiero politico italiano nell'età della Controriforma e barocca. I memoriali redatti dai viaggiatori e le relazioni approntate dai gesuiti offrivano un'immagine complessa dell'impero, che segnò variamente la diffusione del mito cinese nelle scritture politiche italiane del XVI e XVII secolo. La complessità delle descrizioni qualifica soprattutto le caratteristiche della sovranità e i comportamenti tenuti dall'imperatore. A partire da Della entrata della Compagnia di Giesù e Christianità nella Cina è presente in tale letteratura un'immagine molto articolata del governo imperiale: accanto alla forma assolutistica del potere, sono diffuse altre narrazioni che ora riconoscono nel passato una sorta di monarchia feudale, ora vedono le istituzioni vigenti configurare una sorta di governo misto, ora si ergono a critica del dispotismo. Inoltre Matteo Ricci e altri gesuiti, quali Martino Martini e Athanasius Kircher presentarono i mandarini come una sorta di platonici filosofi-re. Seguendo le opere redatte dai gesuiti, teorici della ragion di stato come Giovanni Botero e Lodovico Zuccolo sostennero che la perfezione delle istituzioni politiche e sociali della Cina si poneva come garanzia nei confronti di ogni degenerazione, rendendo l'impero il paradigma dello stato ben ordinato, come scrisse un altro gesuita, $\mathrm{Da}$ niello Bartoli.
\end{abstract}


ABSTRACT. - The present study aims at analyzing the relationship between the image of the Chinese Empire in the writings of travellers and Jesuits and the main themes (Reason of State, Utopianism, Mixed Constitution) of the Italian political thought during the XVI ${ }^{\text {th }}$ and XVII ${ }^{\text {th }}$ centuries. The writings of travellers and Jesuits presented a complex view of the Empire, which impacts in various ways the spread of the Chinese myth in the political treatises of the Counter-Reformation era. The complexity is evident in the description the relations provide of sovereignty and the behaviour of the emperor. From the very beginning of Della entrata della Compagnia di Giesù e Christianità nella Cina is presented a complex image of the Chinese government: along with the absolutist delineation of the imperial power, there are conflicting images that, regarding either a distant past or current government practice, recognized other forms: a feudal style of monarchy, a sort of mixed constitution, or a form of dispotic govetrnment. Matteo Ricci and other Jesuits, as Martino Martini and Athanasius Kircher, presented the Mandarins as a slort of Kings-Philosophers of Plato's Republic. Following the works composed by Jesuits, some authors pf Reason of State and utopist (Giovanni Botero and Ludovico Zuccolo) pointed out that the perfec tion of the Chinese political and social institutions could really preserve the Empire from corruption, an Empire that had to be considered a paradigm of a well ordered state, as another Jesuit, Daniello Bartoli, wrote in his book on China.

Per la cultura italiana tra XVI e XVII secolo la $\mathrm{Cina}^{1}$ non ha rappresentato solamente fonte di meraviglia, atteggiamento che ben dichiarava la propria ascendenza poliana, ${ }^{2}$ e venne manifestato anche dal gesuita Matteo Ricci (1552-1610) ${ }^{3}$ che, presente in Cina dal 1583,

1 Ineludibile rimando, e sostegno all'indagine qui condotta, resta l'importantissima opera di D.F. Lach e E.J. van Kley, Asia in the making of Europe, in particolare il Volume III. A century of advance. Book one: trade, missions, litterature, e Book four: East Asia, Chicago and London, The University of Chicago Press, 1993.

2 Programmatico in tale prospettiva risulta certamente l'incipit del Milione: "Signori imperadori, re, duchi e tutte altre genti che volete sapere le diverse generazioni delle genti e le diversità delle regioni del mondo, leggete questo libro dove le troverrete tutte le grandissime maraviglie e gran diversitadi delle genti d'Erminia, di Persia e di Tarteria, d'India e di molte altre province", cfr. M. Polo, Milione - Le divisament $d u$ monde. Il Milione nelle redazioni toscana e franco-italiana, a cura di G. Ronchi, introduzione di C. Segre, Milano, Arnoldo Mondadori, 1982, p. 3.

3 Sulla figura di questo gesuita maceratese da ultimo si veda R. Po-Chie Hsie, Un gesuita nella Città Proibita. Matteo Ricci (1552-1610), Bologna, il Mulino, 2012, ma importante resta ancora, come sguardo d'insieme, D.E. Mungello, Curious land: Jesuit accomodation and the origin of sinology, Stuttgart, Franz Steiner Verlag Wiesbaden, 1985, su Ricci: pp. 44-73. Per l'esaltazione ricciana della "meraviglia" che la Cina offriva agli occhi dei visitatori occidentali valga come esempio un brano della lettera da lui 
con la sua narrazione Della entrata della Compagnia di Giesù $e$ Christianità nella Cina ${ }^{4}$ avrebbe costruito quella trama di notizie, osservazioni e riflessioni che sarebbero tornate in gran parte degli scritti sul Regno di Mezzo redatti da altri padri della Compagnia. Tuttavia, a ben testimoniare la ricchezza di giudizi contenuti nelle opere di viaggiatori e di missionari, può far fede un brano tratto dai Ragionamenti del mio viaggio intorno al mondo, testo certo estraneo alla fortuna di cui godette l'opera ricciana, in cui il mercante fiorentino Francesco Carletti (1573 o 1574-1636) riportava le sue impressioni sulla Cina, da lui visitata tra il 1594 e il 1606:

Il modo di stampare ed il fare l'artiglieria e polvere [...] sono tanto antiche invenzioni nella Cina che passano migliaia d'anni, e si può senz'alcun dubbio credere che tutte venghino da loro. Ed io concorrerei a dire che non solamente queste, ma ogni altra invenzione di buono

inviata il 24 novembre 1585 all'amico Giulio Fuligatti, ove affermava che la Cina era "la maggior maraviglia che in questo Oriente si ritruova di cose naturali e soprannaturali”, cfr. M. Ricci, Lettere (1580-1609), edizione realizzata sotto la direzione di P. Corradini, prefazione di F. Mignini, a cura di F. D'Arelli, con un saggio di S. Bozzola, Macerata, Quodlibet, 2001, p. 111.

4 Il manoscritto dell'opera ricciana Della entrata della Compagnia di Giesù e Christianità nella Cina, conservato nell'Archivio Romano della Compagnia di Gesù, venne pubblicato per la prima volta all'inizio del XX secolo nelle Opere storiche del $P$. Matteo Ricci S. I., edite a cura del Comitato delle Onoranze Nazionali con prolegomeni, note e tavole di P. Tacchi Venturi S. I., vol. I, Macerata, F. Giorgetti, 1911. Più recentemente il testo è stato riedito, sotto la direzione di P. Corradini e con prefazione di F. Mignini, a cura di M. Del Gatto, Macerata, Quodlibet, 2000. Il testo seguito nel saggio che qui si pubblica è apparso con il titolo: M. Ricci, Descrizione della Cina, prefazione di B. Valli, con un saggio di F. Mignini, Macerata, Quodlibet, 2011, e riproduce, con alcune varianti, il primo capitolo dell'edizione apparsa a Macerata nel 2000. Vale ricordare che l'opera ricciana ebbe una prima e completa traduzione latina con il titolo De Christiana expeditione apud Sinas suscepta a Societate Iesu. Ex P. Matthaei Ricij eiusdem Societatis commentarijs libri $V$ ad S.D.N. Paulum V. In quibus Sinensis regni mores, leges atque instituta \& novae illius Ecclesiae difficillima primordia accurate \& summa fide describuntur. Auctore P. Nicolao Trigautio Belga ex eadem Societate, Augustae Vind. apud Christoph Mangium, 1615 [colophon: Augustae Vindelicorum, apud Christophorum Mangium, 1615]. Non a caso, però, per l'interesse suscitato dalla descrizione del lontano impero, proprio il primo libro venne separatamente dato alle stampe nelle cosiddette "repubblichine" elzeviriane - come le definiva il grande bibliografo ed erudito toscano Antonio Magliabechi (1633-1714) - con il titolo Regni Chinensis descrptio. Ex variis authoribus, Lugd. Batav., Ex offic. Elzeviriana, 1639. 
o di cattivo, di bello o di brutto, fossero venute da quel paese [...]. Abbondano d'ogni cosa e d'ogni arte meccanica, e pulitica [...], e fanno professione di filosofia morale, di matematica, d'astrologia, di medicina e di altre scienze nelle quali si tengono li primi uomini del mondo e non pensano che sia sapere fuora della loro nazione, tenendo tutti gli altri per gente barbara.

Per quanto la meraviglia avesse costituito - a partire dalla Metafisica di Aristotele ${ }^{6}$ - un fortissimo stimolo all'indagine e alla conoscenza, proprio negli scrittori politici italiani fra Cinque e Seicento era stata attitudine intellettuale che frequentemente era stata assunta quale architettura ingressiva alla trattazione dei temi più dibattuti. ${ }^{7}$ Tuttavia,

5 F. Carletti, Ragionamenti [...] sopra le cose da lui vedute ne' suoi viaggi si dell'Indie Occidentali, e Orientali come d'altri paesi, In Firenze, nel Garbo nella Stamperia di Giuseppe Manni per il Carlieri all'insegna di S. Luigi, 1701: l'opera venne infatti pubblicata postuma dal segretario dell'Accademia fiorentina del Cimento, Lorenzo Magalotti (1637-1712) che era già stato editore nel 1697 di altra relazione sulla Cina, come si vedrà alla nota 119. La citazione nel testo è tratta dagli excerpta dell'opera contenuti in M. Guglielminetti (a cura di), Viaggiatori del Seicento, Torino, Utet, 1969, p. 198; per la biografia di Carletti, appartenente a un'antica famiglia di mercanti fiorentini, si veda la voce redazionale Carletti, Francesco, in Dizionario Biografico degli Italiani, vol. XX, Roma, Istituto della Enciclopedia Italiana, 1977, pp. 139-143. Una rapida ricostruzione della composizione e della diffusione dei Ragionamenti si trova in D.F. Lach e E.J. van Kley, Asia in the making of Europe. Volume III. A century of advance. Book one: trade, missions, litterature, cit., p. 388.

6 Aristotele, Metaphysica, 982b.

7 Infatti, la meraviglia è posizione che nei grandi pensatori politici del Cinque e Seicento accompagnava frequentemente la loro scelta di porsi nel cuore del dibattito politico che caratterizzava il loro tempo. Così, in Giovanni Botero (1544-1617) la "maraviglia" accompagnava la sua critica al machiavellismo e al tacitismo allora imperanti: "mi ha recato somma meraviglia il sentire tutto il dì mentovare ragione di Stato, et in cotal maniera citare ora Nicolò Machiavelli, ora Cornelio Tacito: quello perché dà precetti appartenenti al governo et al reggimento de' popoli, questo perché esprime vivamente le arti usate da Tiberio Cesare, e per conseguire, e per conservarsi nell'imperio di Roma", cfr. G. Botero, Della Ragion di Stato, a cura di P. Benedetti e R. Descendre. Introduzione di R. Descendre, Torino, Einaudi, 2016, dedicatoria All'illustrissimo et reverendissimo Signor mio osservandissimo il Signor Volfango Teodorico Arcivescovo e Prencipe di Salczburg, p. 3. Paolo Paruta (1540-1598), uno dei maggiori pensatori politici veneziani di fine Cinquecento, si avvalse di tale formula proprio all'inizio della sua opera più importante ovvero Della perfezzione della vita politica [...], In Venetia, Appresso Domenico Niccolini, 1579 - per dar corso alla costruzione del mito di Venezia attraverso 
l'attenzione da loro riservata nei riguardi dell'impero cinese ha comportato anche altre, e assai meno note, modalità di approccio. Infatti, per gli intellettuali italiani della prima età moderna le indagini sulla Cina, i suoi costumi civili e la sua forma di governo assolsero a due funzioni euristiche distinte, finora mai chiaramente sottolineate. Da un lato, e soprattutto nelle relazioni di viaggiatori e di missionari, alcuni tra i principali concetti politici elaborati dalla tradizione dottrinale occidentale (monarchia assoluta, dispotismo, governo misto, i filosofire) furono usati per rimodulare la diversità dei costumi e la distanza culturale della Cina in un sistema citazionale di facile comprensione per il pubblico di lettori. ${ }^{8}$ Dall'altro lato, invece, il "modello Cina" e la

l'esaltazione dello stato misto, come condizione del raggiungimento della felicità terrena: "Quante volte [...] meco stesso rivolgo nell'animo le varie opinioni degli uomini intorno alla loro felicità, sentomi sorpreso da gran maraviglia considerando onde sia che così diverse strade siano state seguite, e tuttavia si seguano", il brano è letto nell'edizione fornita in G. Benzoni e T. Zanato (a cura di), Storici e politici veneti del Cinquecento e del Seicento, Milano-Napoli, Ricciardi, 1982, p. 623. In Lodovico Zuccolo (1568-1630) la "maraviglia" introduceva addirittura "un ragionamento sull'ottima forma di governo", che tornava a lode della perpetua libertas in cui da sempre la minuscola Repubblica di San Marino aveva inteso rappresentare la sua storia, cfr. L. Zuccolo, Il Belluzzi, overo della Città felice, in Id., Dialoghi, In Venetia, Appresso Marco Ginammi, 1625, p. 165.

8 Invero, proprio la rimodulazione della descrizione della Cina in una trama di rimandi concettuali che tradizionalmente articolavano i discorsi politici della prima età moderna certamente si presentava come metodo adeguato a superare le difficoltà di dare una corretta presentazione del sistema politico di quel lontano impero, difficoltà che con grande consapevolezza il gesuita portoghese Alvaro Semedo (1585 o 1586 - 1658) aveva puntualmente sottolineato: "Lo scrivere di cose remote ha quasi sempre tirato seco l'inconveniente di molti e non piccioli difetti. Di quelli che hanno scritto della China, ho visto alcuni, che lasciando in oblio quasi tutte le verità, solamente si raggirano in cose, che sono dal vero lontane: perché essendo questo regno, \& havendo sempre con ogni studio fuggito la communicatione co' forestieri, conservando per sé le sue cose come proprie con particolarissima cautela, viene in conseguenza che di quella solamente si sa di fuori ciò, che si lascia cadere, come soprabbondanza, nelle falde del paese di Cantone, parte di questo Imperio, alla quale sono pervenuti i Portughesi”, la si legge nella traduzione in italiano apparsa con il titolo Relatione della Grande Monarchia della China del P. Alvaro Semedo portughese della Compagnia di Giesù, Romae, Sumptibus Hermann Scheus, 1643 [colophon: "In Roma, nella Stamperia di Lodovico Grignani, 1643], la citazione è tratta dal Proemio, pp. 1-2. L'opera ebbe una rapida diffusione se il testo originale, con il titolo Imperio de la China. I cultura evangelica en èl, por los Religios de la Compañia de Iesus. [...], era apparso solo l'anno precedente "Impresso por Iuan Sanchez en Madrid. Año de 1642”. Per una prima informazione sull'opera di Semedo, 
sua pur rituale eulogia ebbero la funzione di esemplificare segmenti concettuali costitutivi delle principali dottrine politiche dell'età della Controriforma e barocca, in particolare la ragion di stato e la ricerca della "ottima forma" di governo che aveva il suo culmine nella trattatistica utopica." Conseguentemente, restano sicuramente riduttive sia una stilizzazione della Cina come governo solo dispotico sia, per converso, la sua caratterizzazione a regno della saggezza e della moralità, quale è espressa in Federico Chabod..$^{10}$ Di contro, vale notare che, per quanto differenti, entrambe le modalità di indagine più sopra ricordate costituirono un chiaro tentativo di offrire un'interpretazione della società e del sistema politico cinese non fondata sulla semplice raccolta di osservazioni empiriche più o meno veritiere e più o meno coerentemente esposte, ${ }^{11}$ bensì elaborata a partire da consolidate teorie dell'ordine politico, capaci, cioè, di integrare l'autorappresentazione che quel lontano impero dava del proprio governo, nelle tipologie che connota-

si veda D. E. Mungello, Curious land: Jesuit accomodation and the origin of sinology, cit., pp. 74-90; sulla sua composizione e diffusione il rimando è a D.F. Lach e E.J. van Kley, Asia in the making of Europe. Volume III. A century of advance. Book one: trade, missions, litterature, cit., p. 349: "In the seventeenth century and since, Samedo's China has been esteemed a reliable and comprehensive firsthand account" sulle condizioni in cui l'impero versava alla fine della dinastia Ming.

9 Per la presentazione di tali generi di scrittura politica quali fondamenti costitutivi della trattatistica dell'età della Controriforma si veda P. C. Pissavino, Le forme della conservazione politica: Ragion di Stato e utopia, in C. Vasoli, Le filosofie del Rinascimento, a cura di P. C. Pissavino, Milano, Bruno Mondadori editore, 2002, pp. 552-606.

10 F. Chabod, Storia dell'idea d'Europa, a cura di E. Sestan e A. Saitta, Laterza, Bari $1971^{5}$, p. 63 .

11 Rispetto a molte relazioni che dell'impero cinese avevano fornito in Europa descrizioni incomplete o di seconda mano, con nettezza e giusto orgoglio Matteo Ricci aveva dichiarato il rigore metodologico posto nella propria opera: "mi mossi adesso a raccogliere e disporre in ordine le cose più notabili di quelle che sino dal principio avevo notato in questa materia [...] e se bene di queste stesse materie so che già vanno molti libri in Europa, con tutto ciò, penso, a nessuno serà discaro saperle più tosto da noi - che già trenta anni viviamo in questo regno, discorressimo per le sue nobili e principali provincie, trattiamo continuamente in ambedue le corti [Pechino e Nanchino] con i più principali e grandi magistrati e letterati del regno, parliamo la loro lingua, e imparassimo molto di proposito i loro riti e costumi”, cfr. M. Ricci, Descrizione della Cina, cit., cap. I, Della Causa di scrivere questa Istoria e del modo che in essa si ha da procedere, pp. 23-24. 
vano il pensiero politico italiano. ${ }^{12}$ In questa prospettiva, il saggio che qui si presenta si soffermerà, oltre che su quelle redatte dai gesuiti, sulle opere di alcuni tra i maggiori autori dell'età della Controriforma Giovanni Botero e Lodovico Zuccolo - che ben illustrano una riflessione che già allora aveva saputo cogliere la complessità ma anche la pluralità delle dinamiche di omologazione/differenziazione della realtà planetaria molto prima che Thomas Lauren Friedman intendesse descrivere il mondo come piatto. ${ }^{13}$ Che, poi, la complessità del sistema politico cinese fosse tale da mettere in discussione ogni tentativo di rinserrarne l'interpretazione in un'unica e, alla fine, semplicistica prospettiva d'analisi resta approdo certo più tardo, e la si deve all'elegante penna di Montesquieu (1689-1755) che nel suo capolavoro, Lo spirito delle leggi (1748), si mostrò assai critico nei confronti delle relazioni approntate dai gesuiti:

I nostri missionari ci parlano del vasto impero cinese come di un governo ammirevole che riunisce insieme, nel suo principio fondamentale, il timore, l'onore e la virtù. Io ho dunque posto una vana distinzione quando ho stabilito i principi delle tre forme di governo [monarchia, repubblica, dispotismo]..$^{14}$

\section{LA SOLITUDINE DEL SOVRANO ASSOLUTO, LA FIGURA DEL DESPOTA}

Per il vero, già dagli scritti di viaggiatori e gesuiti emergeva un'immagine complessa, se non addirittura contraddittoria, dell'impero, che verrà variamente a incidere sull'audizione e sulla circolazione del mito cinese nella trattatistica politica italiana dell'età della Controriforma. Vediamo come, proprio prendendo le mosse dalla connotazione degli attributi di sovranità e del comportamento tenuto dall'imperatore.

Ricorrente in tale letteratura era la minuta analisi degli assetti di

12 Sulla costruzione della teoria politica come conoscenza critica dell'ordine di una data società si rimanda a E. Vogelin, Che cos'è la realtà politica?, in Id., Anamnesis. Teoria della storia e della politica, Milano, Giuffrè, pp. 197-269.

13 Th. L. Friedman, The World is Flat: A Brief History of the Twenty-First Century, New York, Farrar, Straus and Giroux, 2005.

14 Ch. de Secondat barone di Montesquieu, Lo spirito delle leggi, a cura di S. Cotta, Torino, Utet, $1996^{3}$, 1. VIII, cap. XXI, Dell'impero cinese, pp. 229-230. 
potere che caratterizzavano la Cina, analisi presente anche nella Relatione della gran Città del Quinsai, E del Re della China di Contugo Contughi. ${ }^{15}$ Anzi, l'importanza che tale analisi assumeva nella Relatione

15 La Relatione - dedicata, come si legge nel titolo, All'Illustrissimo Signore Luigi Gonzaga. Illustriss. Sig. mio - è datata 16 giugno 1584. Le citazioni riportate nel saggio sono tratte dalla redazione presente nella Continuatione del Thesoro politico, nella quale si contengono $i$ Trattati, Discorsi, Relationi, Ragguagli, Instruttioni, di molta importanza per li maneggi, interessi, pretensioni, dipendenze e disegni de' Principi [...]. Raccolta per il Sig. Lodovico Ricci, In Bologna, Presso gli Heredi di Giovanni Rossi, 1603, dove appare come XVIII capitolo, ma lo scritto di Contughi ricorre ovviamente anche in numerosissime altre edizioni del Thesoro politico. Questa Relatione si trova anche in un manoscritto conservato presso la Biblioteca Nazionale di Napoli, cfr. P. Amat di San Filippo, Biografia dei viaggiatori italiani con la bibliografia delle loro opere, contenuta negli Studi bibliografici sulla Storia della Geografia in Italia pubblicati in occasione del III Congresso Geografico Internazionale, Roma, Alla sede della Società Geografica Italiana, 1882, vol. I, p. 354. Sempre P. Amat di San Filippo ricorda come in un'altra edizione del Thesoro, ovvero Philippi Honorii Thesaurus politicus etc. Opus collectum ex italicis tam publicatis, tam mss. variis variorum observationibus et discursibus, accurato cum delectu concinatum et in gratiam politicae sapientiae et linguarum studiosorum nunc latine simul italice editum. Editio postrema, Francofurti, Typis Nicolai Hoffmanni, impensis haeredum Jacobi Fischeri, 1617 et 1618, sia presente, a nome di Giacomo Pantogia, una Relatio de amplissimo Sinarum regno, cfr. P. Amat di San Filippo, Bibliografia dei viaggiatori italiani ordinata cronologicamente ed illustrata, Roma, Coi tipi del Salviucci, 1871, p. 67. In realtà la Relatio del gesuita Diego de Pantoja (1571-1618) - in Cina al seguito di Ricci dal 1599, e con lui ospite per circa un mese nel palazzo imperiale di Pechino, a partire dal 24 gennaio 1601 - era la traduzione latina di una parte della lettera da lui indirizzata a Luis de Guzmán, arcivescovo di Toledo "Pachini VII Idus Martias MDCII". Non solo: la Relatio era già apparsa in Thesoro politico la parte seconda [...], Francofurti, Apud Ioannem Wolffium, Impensis Ioannis Theobaldi Schönwetteri, 1611, e, nello stesso anno e presso gli stessi editori, nella Praxis Prudentiae Politicae pars secunda [...] Opus collectum ex italicis com publicatis tum manuscriptis variis variorum Ambassatorum observationibus \& discursibus [...] a Philippo Honorio. Da quest'ultima edizione saranno tratte le citazioni riportate nel testo. Per la figura del Pantoja, valga ricordare che nelle sue Controversias antiguas y modernas [...] de la gran China, l'arcivescovo domenicano Pedro Fernández Navarrete lo descrisse, con il Ricci, tra i "Gigantes de la Missión de la China", cfr. J.S. Cummings, Two missionary methods in China: Mendicants and Jesuits, saggio riedito in Id., Jesuits and Friar in the spanish expansion to the East, London, Variorum Reprint, 1986, V, p. 76. Sulla composizione e la diffusione della Relatio si vedano le indicazioni contenute in D.F. Lach e E.J. van Kley, Asia in the making of Europe. Volume III. A century of advance. Book one: trade, missions, litterature, specie a p. 320: "His letter is a gem; it provides some of the most interesting and accurate information about China that became available during the first half of the seventeenth century". 
trova conferma nella sua peculiare collocazione editoriale: venne infatti inserita nel Thesoro politico, forse l'opera più significativa di quella sedimentazione della cultura civile italiana ed europea in Trattati, Ragguagli, Istruzioni pertinenti - come recitava il frontespizio - alla perfetta intelligenza della Ragion di Stato et alla intiera cognitione degli interessi e dipendenze de' più gran Prencipi, E Signori del Mondo: insomma si trattava di una sorta di diffusissima e consultatissima enciclopedia di geopolitica, che si collocava agli albori della politica comparata. ${ }^{16}$

Dopo aver ricordato la ricchezza di cui disponeva l'imperatore della Cina, osservando che "è tanta, che il maggior Prencipe de' Christiani non si potria di leggier a tali de i suoi favoriti porre innanzi" ${ }^{17}$ Contughi veniva a indagare minutamente le funzioni e i comportamenti del sovrano. Infatti sottolineava che

Il Re rarissime volte si lascia vedere, \& quelle con grandissima solennità. È superbissimo, non tanto per i molti stati che possiede, quanto per la nobiltà, poiché pretende che sin dal principio del Mondo i suoi Progenitori, sino a lui successivamente, siano stati Re della China, fuori che novantatré anni, nel qual tempo furono tiranneggiati da' Tartari.

La solitudine del sovrano ${ }^{18}$ veniva poi ribadita ricordando come

16 Su questa opera e sulla sua importanza si vedano i saggi di A. E. Baldini, Origine e fortuna del Tesoro Politico alla luce di nuovi documenti dell'Archivio del Sant'Uffizio, Studia Borromaica, 13, 2000, 155-174; S. Testa, Una interpretazione del Thesoro Politico (1589), Nuova Rivista Storica, 85, 2001, 347-362; Id., Alcune riflessioni sul Thesoro Politico, Bibliothèque d'Humanisme et Renaissance, 64, 2002, pp. 679687, e, infine, Id., From the 'Bibliographical Nightmare' to a critical bibliography. Tesori Politici in the British Library and elsewhere in England, eBLJ, 2008, 1, 1-33, che considera la Relatione del Contughi una "another edition of Marco Polo's description of China, with additions taken from other travellers", p. 11.

17 C. Contughi, Relatione della gran Città del Quinsai [...], cit., pp. 212-213.

18 Tale separatezza ritornava più volte nelle prose civili e letterarie di importanti autori italiani dell'età della Controriforma. Si veda, ad esempio, quanto scrisse Tommaso Garzoni (1549-1589), in La Piazza universale di tutte le professioni del mondo, In Venetia, Presso Giovanni Battista Somasco, 1585, di cui, per la citazione, seguiamo però l'edizione curata da P. Cherchi e B. Collina, Torino, Einaudi, 1996, vol. I, p. 552: "S'afferma il loro re essere il maggiore di tutto il mondo, come quello che, fra l'altre grandezze, abita in un palazzo di sette meraviglie cinto, ove di fuori stan sempre alla guardia dieci mila uomini, mutandosi a vicenda". Alessandro Tassoni (1565-1635), per parte sua, coniugava in una sorta di battuta il potere assoluto di cui godeva l'imperatore e la sua separatezza dal 
l'imperatore conducesse la propria esistenza quasi esclusivamente all'interno del suo palazzo: "non s'impaccia d'alcuna cosa, solo se ne vive rinchiuso nel suo Palazzo fra il gran numero di Damigelle, \& Pagi, né ad altra qualità di gente è lecito vederlo, se non come si è detto solamente con solennità". L'imperatore era quindi raffigurato esercitare il suo immenso potere - "L'amministrazione del tutto sta in potestà del Re" ${ }^{19}$ - rendendo ineludibile la sua separatezza:

Se occorre per caso, che il Re voglia intendere da alcuno de' suoi Ministri, ciò che sia quello, che ha da riferirgli, egli sta in un'anticame$\mathrm{ra}, \&$ con una Ciarabottana le dice all'orecchia del secretario principale, che dalla propria anticamera del Re, col medesimo modo gliel'intona, \& il simile osservano gli ambasciatori de' gran Prencipi a quella città mandati. ${ }^{20}$

Tale condizione non impediva, tuttavia, che le sue decisioni si costituissero a fonte di giustizia, così da garantire un sistema politico e sociale ben regolato: infatti "la giustitia che viene immediatamente dal Re non permette, che muoia alcuno, perché dicono non convenirsi alla Clemenza sua", mentre le stesse leggi "con le quali si governano (per quello, che si riferisce) sono giustissime, di modo, che rare volte fra loro occorrono homicidi, o altre violenze di qualunque sorte". ${ }^{21}$

Radicalmente opposto, e decisamente negativo, sarebbe stato invece il giudizio con cui il gesuita bergamasco Giovanni Pietro Maffei (1536-1603) avrebbe suggellato la sua descrizione della Cina contenuta nel libro VI delle sue Historie delle Indie orientali. ${ }^{22}$ Difatti, per Maffei

mondo: "Però se la potenza e lo star separato provano l'essere incorporeo, anche il Re della China sarà incorporeo, che è potentissimo e mai si lascia vedere", si cita da Pensieri e scritti preparatori, a cura di P. Puliatti, Modena, Panini, 1986, p. 779.

19 C. Contughi, Relatione della gran Città del Quinsai [...], cit., p. 209.

20 Ivi, p. 210.

21 Ivi, p. 209.

22 G.P. Maffei, Le historie delle Indie orientali [...]. Tradotte di Latino in lingua Toscana da M. Francesco Serdonati Fiorentino [...], In Venetia, appresso Damian Zenaro, 1589. Si noti che l'edizione originale latina intitolata Historiarum Indicarum libri XVI [...], era stata edita "Florentiae, apud Philippum Iunctam, 1585", l'anno successivo, quindi, alla redazione dello scritto di Contughi. Le Historie di Maffei ebbero larga diffusione e, secondo Federico Chabod, costituirono una delle principali fonti per le Relationi Universali di Botero, che, "convinto effettivamente della bontà dell'opera 
l'amministrazione dello stato così come i costumi dei cinesi svelavano chiaramente, per così dire, un dark side, se non un rovesciamento dialettico dell'eulogia confezionato da Contughi. Sicché, a netta smentita di quanto si leggeva nel Thesoro politico, Maffei dichiarava che

I giudici delle cose capitali con differir più volte i termini della causa sotto specie di clemenza, essercitano gran crudeltà: percioché i rei in tanto, che sempre ve n'ha gran moltitudine, carichi di catene massimamente di notte, e zeppi fra di loro grandemente sono tenuti in prigioni così aspre, e brutte, che molti ogn'anno chiamano la morte prolungata da' Giudici, e non pochi s'ammazzano per se stessi.

Di più, il sistema normativo era ben lontano dalla perfezione attribuitagli dalla Relatione e, anzi, predisponeva il popolo a essere sottoposto a un governo dispotico. Infatti "Le leggi, e gli statuti sono tali, che rendono i popoli più atti alla servitù, \& alla viltà, che alla virtù, \& alla grandezza dell'animo", così che il sovrano poteva depredare a proprio arbitrio i patrimoni dei sudditi:

Il Re con una certa barbara, e sciocca arroganza si chiama padrone del mondo, e figliuolo del cielo. Tiene ufficiali, \& esattori severissimi, con i quali va diminuendo l'havere de privati, e le facultà acquisite con gran sudore, \& vigilanza; \& ha ordinato una dishonesta gabella sopra '1 guadagno delle meretrici, il che si legge esser stato già fatto dall'Imperador Caligola. ${ }^{23}$

Ritratto fosco, quindi, quello tratteggiato nelle Historie: eppure, ad incipit, Maffei non aveva mancato di iterare la retorica del meraviglioso ${ }^{24}$ e di soffermarsi minuziosamente sulla complessa macchina

maffeiana", lo elogiò già nella Ragion di Stato, cfr. F. Chabod, Appendici a "Giovanni Botero", in Id., Scritti sul Rinascimento, Einaudi, Torino 1967, p. 397. Per un primo ragguaglio sulla vita e gli scritti di Maffei se ne veda la voce curata da S. Andretta in Dizionario Biografico degli Italiani, vol. LXVII, Roma, Istituto dell'Enciclopedia Italiana, 2006, pp. 232-234

23 G.P. Maffei (1536-1603), Le historie delle Indie orientali [...], cit., 1. VI, c. $105 \mathrm{v}$. Non si può tuttavia non notare che poche carte prima, sul problema dell'irrogazione della pena capitale Maffei, aveva riportato un giudizio differente, anzi opposto: "Le cause capitali sono prolungate più volte, né si procede nel condannare, se i giudici non hanno veduto, e considerato la causa, si che in tanta moltitudine di gente sono molto pochi quelli, che finiscono la vita per mano del manigoldo", ivi, c. 102v.

24 Ivi, c. 96r: "la bellezza delle Città è meravigliosa", ad esempio. 
burocratica in cui si articolava l'amministrazione dell'impero sia nelle due corti (Pechino e Nanchino) sia nelle quindici province, ${ }^{25}$ fino a descrivere minutamente la rigidissima selezione a cui i magistrati erano sottoposti nel loro percorso di formazione. Infatti, ricordava che, dopo aver superato numerosi esami pertinenti ai grandi temi della politica (" $i$ Censori propongono subito questioni della republica, e del regno, e così delle liti de' privati”), i giovani ritenuti idonei venivano dall'imperatore posti nel "corpo de' Loitij, ${ }^{26}$ che è titolo che si dà a tutti i nobili", ceto da cui

si costuma creare i Giudici, i consiglieri, e' magistrati, né possono per ambitione, o corruttele montare subito a più alti gradi senza haver dato qualche saggio di sé ne luoghi più bassi, ma quando sono provati, e sperimentati ne' luoghi inferiori, sormontano grado per grado a' superiori. ${ }^{27}$

Certo, l'esatta divisione di compiti e uffici tra le varie magistrature e, soprattutto, la pratica degli esami come la lenta progressione che il singolo magistrato poteva incontrare all'interno della macchina burocratica imperiale mostrava un'immagine ben differente dal malcostume italico dei "carichi di salto" 28 che, contraddistinguendo non raramente

25 Ivi, c. 101r-v, ove illustrava come fosse ben organizzato il governo di ciascuna delle quindici province dell'impero, illustrando le attribuzioni dei più alti magistrati: il maggiore, Tutan, "quasi come Vicario del Re è preposto a tutta la provincia [...]. A questo vanno tutte le cose gravi, e di grande importanza; egli decide la maggior parte per se stesso, \& avvisa sovente il Re per lettere di tutto lo stato delle cose. Il secondo luogo dopo costui tiene il Poncasio, il quale ha la cura dell'entrate publiche, e del fisco regio; questo con un gran numero di scrivani e di guardiani tien la cura della Tesoreria, rivede le ragioni, e libri, e distribuisce gli stipendij, che si pagano d'anno in anno, e salari e le mercedi. Seguita poi l'Ancasio, il quale con un consiglio d'huomini eletti, non solamente giudica le differenze, e le liti civili, ma ancora fa i processi delle cose criminali [...]. Di poi l'Aitan governa le cose di guerra, scrive gli esserciti, provvede l'armate, e principalmente ha cura, che i forestieri a poco a poco non penetrino nelle Città, e ne' luoghi fra terra. All'Aitan è soggetto il Luitisio, che tiene l'ultimo luogo, il quale similmente è perito della guerra, \& ad arbitrio dell'Aitan, secondo che richiede il bisogno, va con l'essercito in varie speditioni".

26 "Loitij" in Maffei, "Laotie" in Ricci: è "nome honorifico" per i magistrati di governo, cfr. M. Ricci, Descrizione della Cina, cit., p. 68.

27 G.P. Maffei, Le historie delle Indie orientali [...], cit., cc. 100v-101r.

28 Cfr. P.C. Pissavino, I giovani, $i$ "carichi di salto", e l'ambizione: la competizione 
l'ascesa di giovani patrizi nell'agone politico delle Repubbliche oligarchiche, era percepita come una profonda infrazione all'ordine civile. Tuttavia, la trama di osservazioni e di giudizi espressi da Maffei proponeva, al più, una sorta di anatomia dell'apparato amministrativo imperiale, da cui non sortisce però alcun accenno alle interazioni tra le varie magistrature, così che il lettore potesse ben comprendere in quali mani fosse posto l'effettivo esercizio del potere. Da tale atteggiamento intellettuale si discostò, però, Matteo Ricci, che già in una lettera all'amico Giulio Fuligatti, datata 24 novembre 1585, indicava quale elemento centrale del buon governo imperiale l'assunzione dei funzionari medianti pubblici esami "senza alcun privilegio dovuto alla nobiltà", sicché "quelli dotati di ingegno tutti possono essere grandi". ${ }^{29}$ E proprio attorno alla rilevanza assunta dal ceto dei mandarini nel Regno di Mezzo, Ricci venne a tracciare la propria ricostruzione della microfisica del potere imperiale in Della entrata della Compagnia di Giesù e Christianità nella Cina.

TRA IMPERO FEUDALE, MONARCHIA ASSOLUTA E REPUBBLICA. DiACRONIA E TIPOLOGIA DELlE FORME DI GOVERNO IN DELLA entrata DELLA Compagnia Di Giesù E Christianità nella Cina DI PADRE MATTEO RICCI

Un testo incipitale, una trama di osservazioni che costituiranno, a ben vedere, la traccia per le successive descrizioni dell'impero e del suo sistema politico: questo, in meno parole, è il valore emblematico che apertamente riveste il capitolo VI del primo libro dell'opera ricciana, intitolato appunto Del Governo della Cina. La silhouette che Ricci disegnò del governo nella Cina degli ultimi imperatori Ming annuncia la struttura di un potere burocratico tradizionale di ascendenza weberiana, ${ }^{30} \mathrm{il}$ cui centro - piuttosto che dalla figura dell'imperatore - è

politica nella cultura repubblicana italiana nella prima età moderna, Metabasis. Filosofia e comunicazione. Rivista internazionale di filosofia on line, 7, 2012, $\mathrm{n}^{\circ} 14,1-27$, www.metabasis.it

29 M. Ricci, Lettere (1580-1609), cit., p. 113.

30 Vale ricordare che, secondo Arnold Zingerle, per Max Weber in Economia e società la Cina rappresentava nella storia lo stato patrimoniale sviluppato con maggior 
rappresentato dai letterati (mandarini) che, attraverso una rigida selezione articolata su esami severissimi, venivano a occupare i diversi gradi delle magistrature che controllavano l'impero. ${ }^{31} \mathrm{E}$, sempre attraverso esami, i funzionari erano sottoposti a un sindacato periodico, che sanciva la permanenza nel loro ruolo o la promozione, quando non fossero però degradati o rimossi a causa delle malversazioni compiute nell'esercizio dell'ufficio. ${ }^{32}$ Sicché la centralità che il corpo di magistrati era venu-

coerenza, cfr. A. Zingerle, La burocrazia cinese e il mutamento dinastico nel secolo XVII. Considerazioni sulla percezione della continuità in Martino Martini, in F. Demarchi e R. Scartezzini (a cura di), Martino Martini umanista e scienziato nella Cina del secolo XVII, Atti del Simposio Internazionale su Martino Martini e gli scambi culturali tra Cina e Occidente, Accademia Cinese delle Scienze Sociali, Pechino, 5-6-7 aprile 1994, Trento, Università degli Studi di Trento, 1995, p. 126. Anzi, secondo Zingerle, il confronto sui mezzi di dominio che si sviluppava tra "signore" e "quadro amministrativo" in tutti i tipi di stato patrimoniale trovava in Cina un peculiare compromesso il cui principale pilastro istituzionale era proprio il sistema degli esami. Tuttavia, che tale forma di selezione del funzionariato mettesse a capo alla costituzione di uno stato razionale era esito contestato da Max Weber nelle sue ultime lezioni all'università di Monaco (inverno 1919 - primavera 1920) rifuse nella sua Storia economica. Linee di una storia universale dell'economia e della società, introduzione di C. Trigilia, Donzelli, Roma 1993, pp. 294-295, dove metteva l'accento sulla formazione solo umanistica del mandarino, che "non è minimamente addestrato nell'amministrazione": insomma "uno stato con funzionari di tal fatta è cosa diversa da uno stato occidentale" ovvero razionale. A ben vedere, tale critica era già stata espressa da Diego de Pantoja, pronto ad accusare i mandarini di incapacità nell'amministrazione, di assoluta ignoranza del diritto determinata da una formazione solo umanistico-retorica, e di malversazioni: "complures Mandarinos praeclare excogitare multa, sed cogitata factis non persequi. Quid plura? Administratio pene ethnica, \& plurimis vitiis cumulata. Carent multis legibus: in iure dicundo animi sui sententiam sequuntur; in suis provinciis novas ferunt leges ut libent. Potest enim quis vel ex eo coniicere, non optime hoc regno administrari, quod non quisquis ornate scribere didicit, idem etiam habeat legislatoris prudentiam. Taceo quod saepe lites in rem suam convertunt, ius violant \&, qua licet, rapiunt", cfr. Iacobi Pantogia S. I. De amplissimo Sinarum regno relatio, cit., p. 240.

31 Sul sistema degli esami si veda la lunga presentazione delineata da M. Ricci, Descrizione della Cina, cit., cap. V, Delle arti liberali, scientie e gradi che nella Cina si danno, pp. 56-63.

32 Ivi, cap. VI, Del Governo della Cina, p. 80: "I capi di tutti i magistrati [...] sono obligati di tre in tre anni a comparire tutti insieme personalmente in Pacchino alla Audientia reale, e dar obedientia al Re; nel qual tempo nelle Corti si fa un essame et inquisitione universale di tutti i mandarini della Cina fuora delle Corti, sì di quei che sono obligati a venire alla Corte, come di tutti gli altri, con molto rigore, di come fanno il loro offitio. E conforme a quello che si ritruova di essi o sono nell'offitio o sono abbassati ad altri più bassi o sono totalmente privati o sono castigati senza nessuna remissione o scusa". 
ta ad assumere, agli occhi di Ricci finiva per trasformare la forma di governo, tanto che lo stesso gesuita, quasi riprendendo una indicazione bodiniana, poteva scrivere che "tiene molto del Republico". ${ }^{33}$ Tuttavia, il comportamento e le res gestae dell'imperatore Wanli, regnante al tempo di Ricci, ne ribadiscono piuttosto il carattere dispotico, almeno alla luce della successiva e famosissima caratterizzazione tratteggiatane da Montesquieu. ${ }^{34}$ Semmai, in forma più articolata, si potrebbe affermare, sulla scorta della testimonianza di Ricci, e ricorrendo alla tassonomia delle forme di governo elaborata da Jean Bodin, ${ }^{35}$ che monarchica era la forma stato della Cina (anzi dispotica, almeno per alcuni autori, come più sotto di vedrà) mentre il governo era detenuto da un'aristocrazia di letterati (mandarini), la cui selezione sulla base del merito ubbidiva, però, al principio della libera partecipazione di ogni suddito agli esami. ${ }^{36}$

Seguiamo ora puntualmente la descrizione della statualità imperiale offerta da Ricci non tanto per sottolineare scarti o, più frequentemente, calchi compiuti dalle opere successive, ma piuttosto per comprendere come dalla trama di relazioni che il gesuita seppe costruire tra realtà cinese e strumenti intellettuali europei potè sortire una lezione complessa, capace di stilizzarsi in una assiologia di lunga durata. Vediamo come.

Consapevole che fosse Ricci di quanto "le cose della Cina comunemente sono assai diverse dalle nostre, e $[$ che] questo trattato si fa

33 Ivi, p. 64.

34 Vedi infra nota 61.

35 J. Bodin, I sei libri dello Stato, a cura di M. Isnardi Parente, vol. I, Utet, Torino 1964, 1. II, cap. VII, Della democrazia, p. 659: "Aristotele ha fatto confusione fra il regime di uno Stato e il suo governo [...] il regime può essere una schietta monarchia regia e il governo di tale stato democratico, nel caso che il principe conferisca indiscriminatamente cariche, uffici e benefici a poveri e ricchi, umili e grandi, senza particolari privilegi per alcuno; o una monarchia regia a governo aristocratico, nel caso che il principe conferisca le cariche solo a un ristretto numero di privilegiati”.

36 Condizione, quest'ultima, che veniva puntualmente indicata da Ricci quando osservava la riverenza assoluta che i sudditi tenenvano nei confronti dei loro governatori "ai quali parlano di ginocchioni nelle loro audientie, se bene sappino esser persone che puochi giorni o mesi avanti non erano niente, figliuoli di lavoratori et artegiani di molto bassa sorte", cfr. cap. VI, Del Governo della Cina, p. 80. Tale sistema viene considerato da Filippo Mignini addirittura "una autentica e rigorosa forma di democrazia”, cfr. F. Mignini, Matteo Ricci e la Cina dei Ming, in M. Ricci, Descrizione della Cina, cit., p. 152. 
principalmente per i nostri europei", ${ }^{37}$ era subito pronto, infatti, a rileggerne le forme culturali e politiche entro schemi filosofici ben diffusi, coniando, anzi, una caratterizzazione che avrebbe costituito un autentico cliché, di cui, pur variamente, si sarebbe avvalsa la letteratura gesuitica successiva:

Prima di dire del governo della Cina è necessario che dichiamo qualche cosa delle sue lettere e gradi che in esse si danno, per essere la parte più principale del suo governo, et un modo che sono diversissimi di tutte le altre nationi del mondo; e se di questo regno non si può dire che i filosofi sono Re, almeno con verità si dirrà che i Re sono governati da filosofi. ${ }^{38}$

Pressoché dalle prime pagine della Descrizione sortiva, quindi, un'immagine della soglia di dominio perlomeno composita: accanto alla silhouette assolutistica che Ricci de stylo disegnava al potere imperiale emergevano tratti discrepanti che, o in un lontano passato o nelle prassi di governo vigenti, ne qualificavano altre forme. Così, se netta era la dichiarazione che si legge ad apertura del capitolo VI: "in questo Regno non si usò mai altro che di Governo Monarchico di un suolo Signore, senza aver notitia di altro", ${ }^{39}$ tuttavia la frase successiva tracciava una storia differente, quasi ad articolare i caratteri di una monarchia feudale: "E nel principio, ancorché fusse un solo Re e signore, con tutto vi erano anco molti signori soggetti al signore Universale, sotto varij titoli, come tra noi, di Duchi, Marchesi e Conti. Ma da 1800 anni in qua, fu sempre senza questi stati particolari" ${ }^{40}$ Di più, illustrando le gesta del

37 Ivi, cap. I, Della Causa di scrivere questa Istoria e del modo che in essa si ha da procedere, p. 24

38 Ivi, cap. V, Delle arti liberali, scientie e gradi che nella Cina si danno, p. 48, sicché non resta quindi un caso che nella De Christiana expeditione apud Sinas il termine "letterati" venisse tradotto con "Philosophi".

39 Vale osservare che il testo della De Christiana expeditione apud Sinas resta su questo passo assai più articolato, quasi per ribadire l'impossibilità di ascrivere la Sinensis Reipublicae administratio a ogni altra forma di governo che non fosse quella monocratica: "sola ea gubernatio ad Rempublicam gerendam probata est, quae in unius Imperio Monarchicam sustinet maiestatem, Aristocratiae, porro vel Dimocratiae, vel alterius cuiuslibet Policratiae, vel ipsum nomen apud Sinas est inauditum”, p. 43.

40 M. Ricci, Descrizione della Cina, cit., cap. VI, Del Governo della Cina, p. 64. Vale notare che per Maffei non si dava invece nessun rimando tra le pratiche di governo 
fondatore della dinastia Ming, Ricci faceva emergere comportamenti che parevano riproporre il costume feudale del beneficium:

A gli Compagni del Re Humvu nella conquista del Regno non solo diede grandissime rendite, ma anco grandi titoli come di Duchi, Marchesi e Conti [...] per sé e per i suoi successori et varij privilegi e Capitanie de soldati, ma in tutto soggetti ai publici Magistrati. ${ }^{41}$

Tuttavia, se il passato imperiale declinava ricordi feudali, l'affermazione della monarchia assoluta, per propria parte, si doveva confrontare con la centralità assunta dal ceto dei mandarini. Vediamo secondo Ricci perché il governo della Cina "tiene molto di Republico":

Percioché, se tutto quanto si fa nel governo deve essere approvato dal Re nei publici memoriali che i magistrati gli danno di tutto quello che hanno da fare, con tutto il Re non fa altra cosa che approvare e riprovare quello che gli propongono, e quasi mai fa niente sopra qualche negocio senza essergli proposto prima da' magistrati che hanno cura di quello. E così mai fa nessun favore o grazia, se non gli fusse proposto da' magistrati esser degno e meritevole di tal grazia e favore. ${ }^{42}$

A ben leggere questo brano, tuttavia, resta evidente che la forma

in Cina e il sistema di potere feudale in Occidente: "Niuno, fuori che il Re solo, mette datij, o gravezze; non vi sono, come appresso di noi, né Conti, né Duchi, né Baroni, né Signori d'alcuna maniera. Il Re dà i magistrati, e governi non tanto in luogo di beneficio, o di premio, quanto di fatica, o d'officio", cfr. G.P. Maffei, Le historie delle Indie orientali [...], cit., c. 103v. Né diversamente si legge nelle Relationi Universali di Botero, cfr. infra il testo relativo alla nota 99. Un paragone tra il ruolo svolto dai mandarini e il ceto nobiliare occidentale per il vero ricorre anche nella Relatio di Diego de Pantoja: "Porro quemadmodum nullum est in Sinis huminum genus eruditis atque doctoribus honestius; ita nullum est meliori indole, neque gravioribus magisque ingenuis moribus praeditum. [...] Quod, si ullum est in Sinis hominum genus quod cum magnatibus nostris, quos Titulatos dicimus, equitibus aulicis, conferri possit, hi unus est Mandarinorum ordo: adeo multi inter illos sunt eximia in suis negotiis sustinendi sedulitate, innocentia, \& boni publici”, cfr. Iacobi Pantogia S. I. De amplissimo Sinarum regno relatio, cit., p. 239. Tuttavia, al di là di siffatta eulogia, de Pantoja, come si è visto a nota 30, è fortemente critico nei confronti dell'operato dei mandarini.

41 M. Ricci, Descrizione della Cina, cit., cap. VI, Del Governo della Cina, p. 67. In realtà il fondatore della dinastia Ming, Zhu Yuanzhang (imperatore dal 1368 al 1398), aveva come titolo di regno Hongwu, non Humvu.

$$
42 \text { Ivi, p. } 68 .
$$


"repubblicana" con cui Ricci intendeva rivestire il governo dell'impero era un'enfatizzazione che, denunciata successivamente dalla Cina di Daniello Bartoli, veniva smentita da altri passi della stessa Descrizione. Perché, per il vero, l'imperatore manteneva su tutti gli affari di stato un supremo controllo, perfino sui pochi memoriali che i privati gli facevano pervenire dove, non a caso, scriveva "Tal magistrato essamini questo negocio e mi dia avviso di esso". ${ }^{43}$ In verità, come scriveva Bodin, "Di fronte al re non vi è magistrato che conservi autorità di sorta", aggiungendo che

Il cancelliere [del regno di Francia] va a raccogliere il parere e l'opinione dei principi del sangue, dei più grandi signori, dei pari e dei magistrati, non però per decidere in base ai loro voti ma semplicemente per riportare al re loro parere, ch'egli può a suo arbitrio accettare o respingere. ${ }^{44}$

Di più: quella interazione tra imperatore e ceto dei funzionari che Ricci veniva a enfatizzare e a trasformare nel "governo dei letterati", per il vero era correntemente prescritta nella coeva trattatistica politica italiana. Ad esempio, l'abate Bernardino Baldi (1553-1617) aveva affermato nel suo L'Arciero, overo della felicità del Principe che:

non solamente lodo l'uso de' consiglieri, ma stimo mal pensoso quel Principe $[\ldots .$.$] che si crede, per prudente ch'egli si sia, di poter vedere, e$ prevedere, tutte le cose senza haver bisogno della vista e della perspicacia dei consiglieri [...]. Concludasi dunque che a' Principi siano utilissimi i consiglieri, non perché governino o lui o per lui, ma perché venga accresciuta in lui la virtù di due potenze: il ben consultare e il giudizio. ${ }^{45}$

Né differente era la posizione riportata da Girolamo Frachetta (1558-1620):

Non può il Prencipe, per molto savio che sia, veder tutto da sé solo, la onde ha bisogno di Consiglieri, che l'illuminino somministrandoli

43 Ibidem.

44 J. Bodin, I sei libri dello Stato, cit., 1. II, cap. I, Di tutte le forme di Stato in generale; e se ve ne siano solamente tre o di più, p. 558.

45 B. Baldi, L'Arciero overo la felicità del Principe, in Id., Versi e prose, In Venezia, Appresso Francesco de' Franceschi Senese, 1590, p. 476. 
concetti, \& ragioni. Ma vuol però, uditi gli altrui pareri, riservare a sé le deliberazioni, altrimenti parrebbe più tosto servire a suoi consiglieri, che comandare. ${ }^{46}$

Balza all'occhio che le prescrizioni dettate al loro principe assoluto dai due scrittori politici italiani intendevano scongiurare proprio quell'esito - i re governati da filosofi - su cui invece Ricci aveva voluto tratteggiare la propria immagine del sistema politico del Regno di Mezzo. Del resto come si è visto non si dava nessuna espropriazione degli attributi di sovranità o, weberianamente, dei mezzi oggettivi dell'impresa politica (risorse economiche, militari, logistiche, ecc.) da parte dei mandarini. ${ }^{47}$ Semmai, tra magistrati e imperatore interveniva quel compromesso, secondo Zingerle, ${ }^{48}$ o quel bilanciamento, secondo Mignini ${ }^{49}$ che davvero rendeva peculiari le dinamiche fisiologiche al sistema politico cinese. Quelle fisiologiche, va aggiunto, perché - come si vedrà in seguito - quelle patologiche paiono tragicamente infrangerne l'equilibrio in senso dispotico.

Puntuale invece ritornava la ricostruzione operata da Ricci della organizzazione burocratica dell'impero cinese: a capo di essa stavano sei tribunali o ministeri, "il maggiore e più eminente di tutti gli altri" è quello "de' Magistrati" che si occupava "di distribuire tutti i Magistrati et offitij" e di controllare il corretto comportamento dei funzionari. ${ }^{50} \mathrm{~A}$ seguire, Ricci enumerava il magistrato "degli erarij", quello "delle Cortesie e riti", quello "de' Soldati", quello "delle fabriche" (ovvero degli edifici pubblici) e, infine, quello "de' Castighi, che risponde ai nostri giudici ciminali" ${ }^{51}$ Insomma, quelle che la coeva trattatistica politica italia-

46 G. Frachetta, Il Prencipe [...] distinto in due libri, In Venetia, Appresso Gio. Battista Ciotti, 1599, p. 66 ma 67.

47 Si veda per tale prospettiva M. Weber, La politica come professione, in Id., La scienza come professione. La politica come professione, introduzione di Wolfgang Schluchter, Torino, Edizioni di Comunità, 2001, p. 50.

48 Vedi nota supra nota 30.

49 F. Mignini, Matteo Ricci e la Cina dei Ming, cit., p. 159.

50 M. Ricci, Descrizione della Cina, cit., cap. VI, Del Governo della Cina, p. 70: “in questo Tribunale si fa tutto per ordine, cominciando tutti da' più piccoli officij et andando ascendendo ad altro maggiore, conforme alle leggi e statuti, e conforme alle informationi che hanno del modo che lo fece negli officij precedenti, et abbassando e anco privando degli officij quei che la fecero male".

51 Ivi, pp. 70-71. 
na aveva scandito come "materie di stato", ${ }^{52}$ nella prassi amministrativa dell'impero cinese diventavano le effettive funzioni a cui, con un'evidente accelerazione dei processi organizzativi e di selezione del personale, ineludibilmente doveva sovrantindere il ceto dei mandarini: "Tutte le cose di tutto il regno dipendono da questi sei tribunali, e così in tutte le Provincie e le Città tengono Mandarini e notarij publici a loro rispondenti con grandissima communicatione e subordinatione". ${ }^{53}$ Superiore per autorità a questi sei tribunali, Ricci ne ricordava "un altro, il più alto di tutta la Corte e di tutto il Regno che è di Colao, che sono tre o quattro persone e possono essere sino a sei" ${ }^{54}$ Fondamentalmente il Colao, o Gelao, aveva il ruolo di consiglio del Principe, ma proprio nella presentazione offertane dal gesuita appaiono spie di una qualificazione dispotica del potere imperiale. Vediamo perché:

Questi non tengono offitio particulare, ma sono come Consiglieri del Re, e sono soprintendenti di tutto, i quali ogni giorno entrano nel Palazzo del Re, e anticamente parlavano spesse volte con l'istesso, stavano con lui sedendo e trattando i negocij anco a bocca. Adesso che il Re mai esce alla udientia, solo stanno lì dentro tutto il giorno, et a tutti i memoriali che si danno al Re pongono la spiditione che se gli deve dare, e poi tornano a mandare al Re, il quale o approva o riprova o muta quello che sta scritto e di sua mano la pone nel proprio memoriale per essequirsi..$^{55}$

Appare evidente che la funzione decisionale che il sovrano con-

52 Il letterato milanese Giuliano Goselini, ancor prima di Botero, nella Lettera in materia di stato, inviata il 26 febbraio 1583 a don Luis de Castilla, allora visitatore generale nello Stato di Milano, prendendo le mosse dalla definizione di che cosa dovesse intendersi per materia di stato - ovvero «tutto quello che si propone, si consulta, e si delibera per fondare uno stato, o per conservarlo, e per ampliarlo»-, ne enunciava meticolosamente i fondamenti: la religione, le leggi, i magistrati, "gli ufficii, le arti, e liberali e mecaniche, le adherenze, le protetioni, le leghe, gli essercitii, le armate, i presidi, le ambascerie, le guerre, le paci”, cfr. G. Gosellini, Lettera in materia di Stato, in La seconda parte del Tesoro Politico, In Milano, In Milano Per Girolamo Bordone, e Pietro Martire Locarni compagni, 1601, p. 518. Per un primo ragguaglio bio-bibliografico si veda M.C. Giannini, Gosellini, Giuliano, in Dizionario Biografico degli Italiani, vol. LVIII, Roma, Istituto della Enciclopedia Italiana, 2002, pp. 110-114.

53 M. Ricci, Descrizione della Cina, cit., cap. VI, Del Governo della Cina, p. 71.

54 Ibidem.

55 Ivi, pp. 71-72. 
serva per sé di molto riduce la valenza, per così dire, "repubblicana" ascritta da Ricci al sistema di governo cinese. Non bastasse, tale eminenza del sovano si mostrava anche nei confronti di due magistrature (Choli e Tauli) che assolvevano a funzioni di censura e sindacatura sull'operato di tutte le magistrature e anche sul comportamento dell'imperatore:

Questi doi officij sono per varij usi, dentro e fuori della corte, conforme a quello che il Re comanda, con grande potere; e così sono assai rispettati e temuti. Ma, fra l'altre cose, hanno offitio di sindachi, di avvisare il Re di tutto quanto sanno di male, non solo di tutti i magistrati, grandi e piccoli, ma anco dell'istesso Re e sua Casa reale [...] offitio molto simile a quello degli ephori di Lacedemonia. ${ }^{56}$

Come ben si può leggere, per quanto fossero temuti e rispettati, questi magistrati in prima istanza agivano in un ambito giurisdizionale "conforme a quello che il Re comanda". Il paragone con la figura degli efori, che a Sparta avevano il compito di sorvegliare la condotta del re e delle altre magistrature, introduceva un'ulteriore cautela nei confronti dell'effettivo potere di controllo detenuto dai censori: "questi non hanno altro potere che di parlare e più tosto di scrivere, non potendo far niente se non gli è concesso dal Re. Ma fanno si bene il loro offitio che ci fa maravigliare; percioché mai cessano di parlare o latrare con i loro memoriali". ${ }^{77}$ Che, poi, Ricci avesse voluto esaltarne l'autorità morale e l'indipendenza di giudizio ${ }^{58}$ con la quale riuscirono talvolta a porre rimedio agli arbitri compiuti dal sovrano, è fatto che aggiunge

56 Ivi, p. 72

57 Ibidem. Sulla rilevanza che nel pensiero politico della prima modernità assunse la discussione sull'eforato si veda ora il saggio di S. Testoni Binetti, Efori. Antichi e moderni sul potere di controllo, Soveria Mannelli, Rubbettino, 2013.

58 M. Ricci, Descrizione della Cina, cit., cap. VI, Del Governo della Cina, p. 72, ove si sottolineava come i censori conducessero la loro azione "con molta libertà, interezza e dimostrazione di desiderio di bene comune". Il confronto/scontro tra il potere assoluto dell'imperatore e la rettitudine intellettuale e morale dei censori è posto in assoluta evidenza anche dalla Relatio di Pantoja: "Nam etsi summam ille [rex] rerum omnium potestatem habeat, parique, quum libuerit, in maximum quemvis Mandarinorum, atque in postremum quemque hominem dominatu utatur: extiterunt tamen per hos annos non pauci, qui insigni loquendi libertate excellentique animi scriptis ad eum litteris quae ab omnibus legi possent [...] vitia eius coarguerent", cfr. Iacobi Pantogia S. I. De amplissimo Sinarum regno relatio, cit., p. 239. 
solo prove per configurare il potere dell'imperatore come dispotico, pronto infatti a castigare "molto atrocemente" i censori:

E, se bene il Re si adira molte volte con loro e, perché toccano molto al vivo in sua persona et in magistarti grandi, e gli priva di ogni offitio e rendita, o abbassa o castiga molto atrocemente, con tutto ciò loro non cessano di una e più volte tornare a riprendere la stessa cosa, mentre gli dura l'offitio, e mentre non si dà qualche remedio al male che vedono farsi al buon governo. ${ }^{59}$

Tuttavia, Ricci era perentorio nel ribadire che "tutto il regno si governa per letterati, come di sopra ho detto, et in essi sta il vero e misto imperio, ai quali sono soggetti tutti i soldati e i loro capitani”, anche se poco oltre ribadiva come il sovrano stesse a capo della catena di comando, ricordando: "La grande subordinazione che un magistrato inferiore tiene al suo superiore, e quelli fuori della Corte a i Curiali e tutti insieme al loro Re" ${ }^{60}$

Di più: esisteva, comunque, una sorta di dark side di questa meravigliosa macchina di governo, che Ricci non si esimeva dal condannare: ovvero la nefasta influenza che gli eunuchi esercitavano nella corte (infatti "dentro e fuora il palazzo" si commettevano "molte ingiustizie e crudeltà senza potersegli resistere o por rimedio") e sull'imperatore, a tal punto da infrangere l'immagine di "governo di letterati", che inizialmente aveva inteso costruire, e trasformarla nel governo degli eunuchi:

vennero questi [gli eunuchi] a tenere tanto podere nel palazzo, che molti pigliorno questo mezzo per stare più appresso al Re; et a questo tempo a tale che tutti i servitori, Consiglieri e più amici del Re, e che si può dire governano questo regno, sono eunuchi, de' quali stanno nel Palazzo dieci milia. ${ }^{61}$

59 M. Ricci, Descrizione della Cina, cit., cap. VI, Del Governo della Cina, p. 72

60 Ivi, p. 79. Anche questa caratteristica era ricordata come distintiva degli istituti imperiali cinesi rispetto a quelli europei "in quibus - come si legge nella De Christiana expeditione [...], cit., p. 58 - ab Europaeis Sinae discrepant”.

61 Di più, "questi eunuchi soli parlano con il Re, e né virtù, né autorità, né animo tengono per parlargli altra cosa se non quello a che vedono più inclinato quello che pensano essere il suo Dio", M. Ricci, Descrizione della Cina, cit., cap. IX, Delle Superstitioni et d'alcuni abusi della Cina, p. 116. Non si può certo affermare che tale descrizione non si conformi con l'immagine, per così dire, standardizzata del governo 
Amaramente poi segnalava anche le malversazioni e le crudeltà compiute dai mandarini: "E se il Capo di questo governo sta di questa guisa, facil sarà pensare quali sieno i suoi membri che sono i magistrati e mandarini", pronti "a loro voglia" a fustigare a morte i sudditi:

Con questo potere sono molto temuti da tutti e fanno molte ingiustizie cavandogli dalle mani danari e quello che vogliono; perché avendo i poveri sudditi paura di questo castigo sì vergognoso e crudele e della morte che di qui gli può venire, danno tutto quanto tengono per liberarsi dalle loro mani. ${ }^{62}$

Sicché, l'immagine del mirabile governo della Cina, che con tanta cura era stata elaborata nelle pagine iniziali del capitolo VI, veniva infranta dalla truculenza dei comportamenti dispotici, così come offuscata ne era l'ascrizione alla forma repubblicana, che più correttamente Nicolaus Trigaut aveva ricapitolato nella dottrina del governo misto, della quale si era avvalso il pensiero politico italiano ed europeo della prima età moderna per descrivere regimi politici a organizzazione complessa, quale, appunto, quella che caratterizzava il Regno di Mezzo. ${ }^{63}$

\section{DIFFERENTI IMMAGINI DEL POTERE: GOVERNO MISTO O I PLATONICI FILOSOFI-RE?}

Infatti, la descrizione dell'impero cinese contenuta nella $D e$

dispotico che sarebbe stata confezionata da Montesquieu per i "principi d'Oriente". Del
resto, lo stesso Ricci legava l'uso degli eunuchi all'immagine classica del dispotismo orien-
tale osservando che i cinesi, "allo stesso modo che fanno i Turchi", castravano i piccoli
"per porli poi al servizio del Re", ivi, p. 115. Montesquieu avrebbe sostenuto che gli eunu-
chi "hanno infiacchito il cuore e lo spirito [del monarca]", aggiungendo che "Più l'impe-
ro è esteso, più s'ingrandisce il serraglio; e più quindi i piaceri inebriano il principe. Così
in siffatti Stati, il sovrano più ha popoli da governare, meno pensa al governo; più gli affari
sono importanti, meno se ne discute", cfr. Ch. de Secondat barone di Montesquieu, Lo
spirito delle leggi, cit., vol. I, l. II, cap. V, Delle leggi relative alla natura dello Stato dispotico,
pp. 81-82. Non diversamente, Ricci aveva descritto l'imperatore Wanli allontanarsi pro-
gressivamente dalla pratica diretta di governo, ad esempio, non ricevendo più in udienza
il Colao, cfr. M. Ricci, Descrizione della Cina, cit., cap. VI, Del Governo della Cina, p. 72.
62 Ivi, pp. 116-117.
63 Tra le più recenti opere dedicate a tale importantissima formula del linguaggio 
Christiana expeditione si allontanava dal dettato del manoscritto ricciano facendo ricorso alla strumentazione concettuale del governo misto per qualificare l'interazione di poteri che legava la monarchia assoluta e il ceto dei mandarini descritto come aristocrazia:

Etsi dixerim initio, Monarchicam esse huius regni administrandi rationem, tamen e dictis facile constat, \& e dicendis constabit, Aristocratiae non parum admisceri. Quamquam enim omne, quod a Magistratibus statuitur, necesse sit, ab Rege libellis ei datis confirmari; nihil tamen ulla in re decernit prius, quam a Magistratibus ante solicitetur aut moneatur. ${ }^{64}$

Tuttavia, a ben guardare, il governo misto descritto da Trigaut, mentre non dava luogo alla partecipazione, nella soglia di dominio, di differenti istanze e sociali (popolari e aristocratiche che fossero), si apriva invece alla ricomposizione di ascendenza platonica di sapere e potere, esito che, se non si poneva a compiuta alternativa all'assolutismo dell'imperatore, diveniva condizione per criticare e moderare, come si è visto, l'arbitrio e la crudeltà da lui esercitate. Infatti, se per Trigaut l'aristocrazia mandarina aveva dato vita a un governo misto, nell'opera di un gesuita dalla cultura enciclopedica, quale fu Athanasius Kircher (1601-1680) ${ }^{65}$ si

politico si veda, per cura di M. Gaille-Nikodimov, Le Gowvernement mixte. De l'idéal politique au monstre constitutional en Europe (XIII -XVII siècle), Saint-Étienne, Publications de l'Université de Saint-Étienne, 2005.

64 De Christiana expeditione apud Sinas suscepta a Societate Iesu, cit., p. 47.

65 A. Kircher, China monumentis, qui sacris qua profanis, nec non variis naturae \& artis spectaculis, aliarumque rerum memorabilium argumentis illustrata, auspiciis Leopoldi Primi [...], Amstelodami, Apud Jacobum Meurs, 1667, Pars IV, De Politica Sinarum, p. 166: "Rex Monarchiae totius absolutissimus \& Caput \& Dominus est, totumque imperium ita ab Imperatore regitur, ut nulli quid ipso inconsulto liceat”. Per una articolata ricostruzione della biografia intellettuale di questo gesuita dalla cultura enciclopedica si vedano da ultimi gli studi di J.E. Fletcher, $A$ study of the life and works of Athanasius Kircher, 'Germanus incredibilis'. With a selection of his unpublished corrispondence and an annotated translation of his autobiography, Edited for publication by E. Fletcher, Leiden-Boston, Brill, 2011, e di J. Godwin, Athanasius Kircher's theatre of the world. His life, works and the search for universal knowledge, London, Thames and Hudson, 2015². Sull'importanza della China illustrata si veda anche D.E. Mungello, Curious land: Jesuit accomodation and the origin of sinology, cit., soprattutto le pp. 134 173; e, soprattutto, D.F. Lach e E.J. van Kley, Asia in the making of Europe. Volume III. A century of advance. Book one: trade, missions, litterature, soprattutto pp. 485-486 (ove la China illustrata è detto "one of the century's most influential treatises on China"). 
assisteva alla compiuta assimilazione del loro ruolo a quello dei filosofi-re che Platone (Respublica, 473d) aveva posto a capo della sua città ideale. Vale osservare che l'una e l'altra formula (governo misto e filosofi-re) nella coeva tradizione del pensiero politico erano stati segmenti fondativi per operare, pur seguendo prospettive differenti, peculiari idealizzazioni di sistemi politici se non un richiamo a utopia. Al governo misto, infatti, si ispiravano sia le presentazioni del regime veneziano offerte da Gaspare Contarini (La Republica, e i Magistrati di Vinegia [...], nella fortunata versione italiana "Nuovamente corretta e stampata", apparsa "In Vinegia, Per Domenico Giglio, 1564") e da Paolo Paruta (Della Perfettione della vita politica [...]. Libri tre, In Venetia, Appreso Domenico Nicolini, 1599). D'altro lato, i filosofi-re - come le tre primalità dell'essere (Pon, Sin, Mor) elaborate dalla filosofia di Tommaso Campanella - erano addirittura posti a guida della sua Citta del Sole.

Anche Kircher non disconosceva affatto la struttura assolutistica del potere che, prima facie, dominava l'impero, tuttavia ne metteva chiaramente in luce la peculiare articolazione dei saperi che la strutturavano:

Habet \& Rex duo consilii Assessores, quos Calaos vocant, hi uti consummata politices scientia instructi sunt, ita quoque primo post Regem loco habentur. Habet et variorum graduum praefectos, quos Mandarinos vocant, omnes literis \& scientia conspicuos, quibus urbium, publicorumque negotiorum curae $\&$ negotia commissa sunt.

Sicché l'impero cinese diveniva compiuta realizzazione di quella respublica a capo della quale Platone aveva posto appunto i filosofi:

ita ut totum Regnum eo fere modo, quom Platonica Respublica a solis Literatis administrentur, adeoque Platonis votum in Sinensi Monarchia completum videatur, felix nimirum Regnum id futurum, in quo Rex philosopharetur, vel Philosophus imperitaret.

Di più, a stabilizzare il sistema politico cinese concorreva una formidabile amministrazione della giustizia: "Summus Judicum in sceleratis puniendis rigor, \& severitas: quae quidem in tanto Imperio originem suam non habent, nisi ex optimis legibus ad pacem, \& tranquillitatem Regni conservandam, stabilitis". ${ }^{66}$

66 A. Kircher, China monumentis [...], cit., p. 166. 
Che poi la finalità prima del sistema normativo e delle prassi di governo che animavano l'impero orientale si realizzasse nella conservazione della "pax, \& tranquillitas Regni” era esito, invero, già descritto da Ricci quando aveva lodato le leggi emanate dall'imperatore Humwu nelle quali, appunto, "la principal mira [...] fu la pace e quiete del regno, e perpetuare lo stato ne' suoi successori" ${ }^{67} \mathrm{Ma}$, a ben guardare, era esito che omologava la descrizione del "modello cinese" alla elaborazione, a livello dottrinale, delle logiche di governo perseguite dalla monarchia iberica in Italia. Infatti pax y quietitud erano i valori a cui l'ideologia degli Asburgo ricorreva al fine di giustificare e conservare il proprio dominio nella Penisola. Non solo: quel che resta importante notare è che la costruzione dell'ideologia della paz y quietitud avveniva nelle opere di letterati filo-asburgici facendo ricorso a tasselli concettuali che sarebbero tornati immutati nelle pagine dei gesuiti. Si legga, ad esempio, quanto scriveva Luca Contile (1505-1574) in una sua lettera dedicatoria All'Invittissimo e Potentissimo Filippo d'Austria Catholico Re di Spagna: "La Quiete (dico) e la tranquillità veramente in quella città si fruiscono dove la Giustitia si conserva per il publico e privato bisogno". ${ }^{68}$

Ancora un gesuita, Martino Martini (1614-1661), connotava de stylo la forma assoluta di governo della Cina, ricordando, inoltre, come la separatezza dell'imperatore in nulla ostava al controllo oculatissimo che poteva esercitare sull'immenso territorio:

67 M. Ricci, Descrizione della Cina, cit., cap. VI, Del Governo della Cina, p. 66. Che la conservazione della pace e della tranquillità fosse obiettivo della legislazione cinese era calco a tal punto iterato da tornare ancora in Montesquieu: "I legislatori cinesi ebbero come scopo principale del governo la tranquillità dell'impero", cfr. Ch. de Secondat barone di Montesquieu, Lo spirito delle leggi, cit., vol. I, 1. XIX, cap. XIX, p. 505.

68 L. Contile, Ragionamento sopra la proprietà delle imprese con le particolari de gli Accademici Affidati et con le interpretationi et croniche, in Pavia, l'anno 1574 (colophon: "nella Inclita città di Pavia, Appresso Girolamo Bartoli"). Sulla biografia di questo intellettuale senese che era stato al servizio di Ferrante Gonzaga, governatore dello Stato di Milano per Carlo V, e quindi ben addentro all'ideologia imperiale degli Asburgo, si veda C. Mutini, Contile, Luca, in Dizionario Biografico degli Italiani, vol. XXVI, Roma, Istituto della Enciclopedia Italiana, 1983, pp. 495-502. Sull'ideologia della paz y quietitud a cui si informava il dominio spagnolo nell'Italia dell'età della Controriforma, cfr. P. Pissavino, Per un' immagine sistemica del Milanese spagnolo. Lo Stato di Milano come arena di potere, in P. Pissavino e G. Signorotto (a cura di), Lombardia Borromaica Lombardia Spagnola 1554-1659, Roma, Bulzoni, 1995, pp. 163-231. 
Rex ipse vitae ac bonorum omnium absolutissimus est dominus, ipse unus omnium omnino caput, adeoque regnum Sinicum jure absolutissimum Monarchicum dicendum. Illud praeterea in hoc regimine rarum, quod licet Imperator palatio numquam, aut vix umquam exeat, tamen totius Regni statum, ac omnium praefectorum probe novit, propterea annis singulis ad singulas Provincias, visitatorem mittit, ampla supra omnes praefectos auctoritate munitum, tamquam legatum suum; hic Provinciam suam visitat, \& praefectorum omnium virtutes, ac vitia accurate examinat, ut in Regiam reversus, de singulis Imperatorem scripto datis informationibus edoceat. ${ }^{69}$

Anche Martini sposava, come già Ricci, l'ideologia di dominio e controllo attuata dal ceto dei letterati/mandarini a tal punto da sottolinearne la continuità sotto la conquista operata dai Manciù, i quali "stylum Politices Sinicae modumque gubernationis omnino non mutarunt [...] imo sinicis Philosophis, ut antea, regendas urbes ac Provincias concesserunt $[. .$.$] examina Litteratorum, ut antea, relique-$ runt" ${ }^{70}$

Se la pagina del De Bello Tartarico testimoniava come i mandarini costituissero il più efficica apparato di conservazione della statualità imperiale, vale anche osservare come l'immagine di tale ceto, tratteggiata dalla Sinarum Imperii geographica descriptio, ben si differenziava da quella lasciataci da Ricci o da Kircher perché rileggeva la proposta platonica dei filosofi-re nella silhouette, adusa a un uomo di Chiesa, dell'organizzazione propria agli ordini religiosi della Cattolicità. Stabilizzate che fossero le dinamiche di esercizio del potere imperiale,

69 M. Martini, Atlas Extremae Asiae, sive Sinarum Imperii geograpbica descriptio, in W.J. Blaeu, Theatrum orbis terrarum, sive novus atlas, Amsterdami, apud Iannem Blaeu, 1650-1654, p. 11. L'opera è stata recentemente ripubblicata nel volume III, 2 degli Opera omnia di Martino Martini, editi a Trento 1998-2002, a cura di F. Demarchi con la direzione scientifica di G. Bertuccioli. Sulla vita e sull'opera del gesuita, oltre ai saggi raccolti nei già citati Atti del Simposio Martino Martini umanista e scienziato nella Cina del secolo XVII, si veda ora F. Masini, Martini, Martino, in Dizionario Biografico degli Italiani, vol. LXXI, Roma, Istituto della Enciclopedia Italiana, 2008, pp. 244-246. Sulla figura e l'opera di Martini si vedano anche D.E. Mungello, Curious land: Jesuit accomodation and the origin of sinology, cit., soprattutto le pp. 106-133; e, soprattutto, D.F. Lach e E.J. van Kley, Asia in the making of Europe. Volume III. A century of advance. Book one: trade, missions, litterature, pp. 381-382 e 480-481.

70 M. Martini, De Bello Tartarico Historia, pubblicata in appendice all'Atlas, cit., p. 15. 
non a caso venivano assimilate alle regole che presiedevano alla vita delle comunità religiose nella Chiesa controriformistica:

plane Sinica respublica bene constituti Religiosi ordinis regimen imitatus, nec mirum, cum in eo perficiendo a tot millibus annorum Sinici philosophi insudarint, nulli alteri rei fere magis studentes: vero hoc Regnum felix vocarent ille qui optabat rempublicam, in qua vel Rex philosopharetur, vel Philosophus imperaret. ${ }^{71}$

Che poi tale assimilazione tra regole degli ordini religiosi e inalterabili prassi di governo segnalasse la perfezione pressoché utopica degli assetti del potere imperiale, è considerazione che trova conferma, seppur e converso, in uno dei maggiori autori politici nell'Italia del Seicento, Lodovico Zuccolo, attento a paragonare le forme del vivere prescritte da Thomas More per la sua Utopia all'asfittica esistenza che si conduce in un "grandissimo Monasterio". ${ }^{72}$

\section{BOTERO: L'IDEOLOGIA DELLA CONSERVAZIONE E IL GOVERNO DISPOTICO}

Rispetto alla minuziosa architettura narrativa che verrà offerta dalle relazioni di Cina redatte dai gesuiti, va subito detto che altra era la prospettiva dottrinale perseguita da Botero. Certo anche lui aveva offerto nelle sue opere un'immagine, per così dire, completa dell'Impero di Mezzo: dalle dimensioni territoriali alle risorse naturali, dalle attività economiche alla forma di governo. In verità, la descrizione più estesa, contenuta nelle Relazioni Universali, ${ }^{73}$ si presentava come

71 Id., Atlas Extremae Asiae, sive Sinarum Imperii geographica descriptio, cit., p. 11

72 L. Zuccolo, L'Aromatario, overo della Republica d'Utopia, in Id., Dialogbi, cit., p. 260. Vale ricordare come il paragone tra città utopica e monastero sarebbe occorso anche nella pagina di un altro grande pensatore politico italiano, Tommaso Campanella (1568-1639), pronto a ricordare nella Quaestio quarta de Optima republica che i critici della sua Città del Sole obbiettavano che "quanti vivono in una simile repubblica, vivono come monaci”, cfr. T. Campanella, La Città del Sole e Questione quarta sull'ottima Repubblica, a cura di G. Ernst, Milano, Biblioteca Universale Rizzoli, 1996, p. 103.

73 Le Relationi Universali, pubblicate per la prima volta nel 1590, costituiscono una sorta di summa geopolitica e geoeconomica: per una prima informazione su tale opera e, più in generale, sulla biografia intellettuale dell'Autore, si veda L. Firpo, 
una sorta di sistematizzazione delle numerose osservazioni che appaiono nelle opere precedenti, ovvero Delle cause della grandezza delle città libri III (In Roma, Appresso Giovanni Martinelli, 1588) e Della Ragion di Stato libri X (In Venetia, Appresso i Gioliti, 1589), ma proprio per questo motivo vi ribadiva, come si vedrà, la precipua istanza politica, ovvero l'attenta elaborazione di una dottrina generale della conservazione dei sistemi politici. ${ }^{74}$

In primo luogo vale notare che l'analisi condotta da Botero era stata volta a dissipare ogni fascinazione dettata dal meraviglioso ${ }^{75} \mathrm{e}$, piuttosto, veniva ad avanzare argomentazioni razionali per ribadire quanto fosse veritiero il computo delle risorse economiche di cui poteva disporre l'imperatore:

Si tiene che il re della China abbia più di cento millioni d'oro d'entrata, ${ }^{76}$ il che, sebbene pare incredibile ad alcuno, io lo stimo verissimo,

Botero, Giovanni, in Dizionario Biografico degli Italiani, vol. XIII, Roma, Istituto della Enciclopedia Italiana, Roma 1971, pp. 352-362. Si veda altresì il volume A.E. Baldini (a cura di), Botero e la 'Ragion di Stato', Atti del convegno in memoria di Luigi Firpo (Torino 8-10 marzo 1990), Firenze, Olschki, 1992.

74 Come aveva riconosciuto già R. De Mattei, Contenuto ed origini dell'utopia cittadina nel Seicento, Rivista Internazionale di Filosofia del Diritto, 9, 1929, p. 421: "Conservazione è la parola d'ordine come garanzia di quella perfezione che una espansione potrebbe compromettere". Sull'ideologia della conservazione sviluppata nella trattatistica cinque e seicentesca - oltre al già citato saggio di P.C. Pissavino, Le forme della conservazione politica: Ragion di Stato e utopia - si vedano gli scritti di G. Borrelli, Ragion di Stato e Leviatano. Conservazione e scambio alle origini della modernità politica, Bologna, il Mulino, 1993, e 'Non far novità'. Alle radici della cultura italiana della conservazione politica, Napoli, Bibliopolis, 2000.

75 Infatti, alla descrizione delle maggiori città della Cina Botero aveva fatto seguire tale considerazione: "Né debbono queste cose parere ad alcuno incredibili, perché oltre che le relazioni di Marco Polo affermano cose anco maggiori, sono oggidì tanto chiare per gli avvisi, che n'abbiamo continuamente da persone e secolari e religiose e da tutta la nazione portoghese, che il negarle sarebbe un mostrarsi scemo anziché giudicioso", cfr. G. Botero, Delle cause della grandezza delle città, in Id., Della Ragion di Stato con Tre libri delle cause della grandezza delle città, due "Aggiunte" e un "Discorso" sulla popolazione di Roma, a cura di L. Firpo, Utet, Torino 1948, 1. II, cap. XII, Della residenza del prencipe, p. 390. L'operetta nel 2016 ha avuto ben due edizioni, presso Aragno editore a Torino, a cura di Claudia Oreglia e con un saggio di Luigi Firpo, e presso Viella, Roma, a cura di Romain Descendre.

76 Il computo delle assai cospicue entrate di cui godeva l'impero costituì sempre motivo d'interesse e anzi come oggetto di indagine ritornava nella maggior parte delle 
supposto che sia vero quel che si scrive della grandezza dell'imperio, della fertilità del paese, della ricchezza delle minere, dell'innumerabile moltitudine degli artegiani e de' mercanti, della commodità delle strade lastricate per tutto il regno, dell'opportunità de' fiumi navigabili, del numero, grandezza, frequenza delle città, della sottigliezza degli ingegni, della industria dei popoli, che non lasciano perdere un palmo di terra, né perire un'oncia di materia, per vile ch'ella si sia. ${ }^{77}$

In Delle cause della grandezza delle città la considerazione per la potenza dell'impero svelava, quindi, l'importanza delle risorse, per così dire, 'infrastrutturali' ed economiche che la sostenevano, qualificando, anzi, la speciale cura che i sovrani mostravano per la conservazione del proprio stato:

perché con ispesa incredibile [gli imperatori] hanno selicato tutte le strade di quel famosissimo regno, fatto ponti di pietra sopra fiumi immensi $[\ldots]$ lastricate con pietre vive le pianure, sì che, non meno d'inverno che d'estate si camina agevolmente a piedi ed a cavallo e vi si conducono facilmente le mercatanzie e su carri e su bestie da soma. ${ }^{78}$

Ed è in tale prospettiva che va letto, nel capitolo XII, un breve

relazioni di missionari e viaggiatori. Maffei, ad esempio, avanzava qualche perplessità sulla veracità delle stime che erano state elaborate: "Quanto a tesori, e masse ragunate dell'oro, e dell'argento si raccontano cose meravigliose, e vi sono quelli, che riducendo il conto alla ragione della moneta nostra, affermano di certo, che il Re ha d'entrate ogn' anno cento venti milioni d'oro, quanto a pena lasciò morendo nella camera Imperiale l'Imperador Vespasiano, il quale fu diligentissimo nell'accumular tesoro, somma di vero molto grande, e la quale meritamente porta seco qualche difficoltà ad esser creduta", cfr. G.P. Maffei, Le historie delle Indie orientali [...]., cit., c. 103v. Tale perplessità non era affatto condivisa da Matteo Ricci, pronto, anzi, a sostenere che "Le rendite, tributi e gabelle del Regno [...] montano senza dubio più di cento e cinquanta milioni l'anno", cfr. M. Ricci, Descrizione della Cina, cit., cap. VI, Del Governo della Cina, p. 69. A tale ammontare accondiscende anche Kircher: "Reditus vero annuos 150000 milliones aureorum nostratum publica aestimatione, pertigisse; hodierno vero Tartarorum Imperio computatis una redituum ex Tartaricis Regnis proventibus multum etiam excedere certum est”, cfr. A. Kircher, China monumentis [...], cit., p. 167.

77 G. Botero, Della Ragion di Stato, 1. VII, cap. X, Che nel tesoreggiare non si deve procedere in infinito, in Id., Della Ragion di Stato con Tre libri delle cause della grandezza delle città, due "Aggiunte" e un "Discorso" sulla popolazione di Roma, cit., p. 237.

78 Id., Tre libri delle cause della grandezza delle città, 1. II, cap. IX, Dell'avere in sua possanza qualche mercatanzia di momento, ivi, p. 380. 
ritratto complessivo della Cina che Botero redigeva a dichiarata eulogia del lontano paese:

Non è mai stato un regno (parlo de' regni uniti e per così dire d'un pezzo) né più grande, né più popolato, né più ricco, né più dovizioso d'ogni bene della China, né che si sia per più secoli mantenuto; quindi nasce che le città, nelle quali i suoi re han fatto residenza, sono le maggiori che mai siano state al mondo, e queste sono tre: Suntien, Anchin e Panchin. ${ }^{79}$

Questo brano, tuttavia, non costituisce "una esaltazione generica della Cina" - come pure è stato sostenuto ${ }^{80}$ - ma è piuttosto testimonianza evidente della volontà di ricapitolare il carattere di alterità che contrassegnava, agli occhi degli intellettuali italiani, il Celeste Impero in una trama di riflessioni che ne riportava l'esemplarità entro la logica della conservazione politica. Tale logica era, in assoluto, il valore fondativo della cultura civile italiana tra Cinque e Seicento, e Botero seppe declinarne, nelle Cause della grandezza delle Città, le precondizioni materiali ed economiche e, nella Ragion di Stato, le regole prudenziali di stabilizzazione. Così nelle Cause della grandezza delle Città da subito dichiarava la ricchezza e la varietà dei prodotti facendole dipendere dalla posizione geografica:

Or la China è la più oriental parte che si sappia della terra, onde ella si gode di tutte quelle perfezioni che s'attribuiscono all'oriente: ${ }^{81} \mathrm{e}$ prima

79 Ivi, l. II, cap. XII, Della residenza del principe, p. 388.

80 S. Zoli, La Cina e l'età dell'Illuminismo in Italia, Patron, Bologna 1974, p. 232.

81 Vale ricordare che tale considerazione era topos presente nella letteratura politica di fine Cinquecento, si legga, ad esempio, una simile posizione in una delle opere più importanti di quel periodo, ovvero J. Bodin, I sei libri dello Stato, a cura di M. Isnardi Parente e D. Quaglioni, Utet, Torino 1997, 1. V, cap. I, Del metodo da seguirsi per adattare la forma dello Stato alla diversa natura degli nomini; e del modo di conoscere la natura dei popoli, vol. III, pp. 70-71: "per giudizio comune di tutti gli antichi consta che i popoli orientali sono più miti, cortesi, affabili e ingegnosi dei popoli occidentali e meno bellicosi. [...]. Gli Spagnoli hanno notato, che i popoli della Cina, i più orientali che ci siano, sono gli uomini più ingegnosi e cortesi del mondo". Come è noto, l'opera di Bodin, pubblicata in francese per la prima volta nel 1576, ebbe circolazione in Italia anche grazie alla traduzione apparsa a Genova con il titolo I sei libri della Republica del Signor Giovanni Bodino, tradotti di lingua francese nell'italiana da Lorenzo Conti [...], In Genova, Appresso Girolamo Bartoli, 1588, p. 446. 
l'aere, del quale non è cosa nessuna che più importi alla vita, aiutato dalla vicinanza del mare [...] vi è generalmente temperato. Il paese poi è generalmente piano e di natura attissima alla produzione di ogni delicatezza, nonché delle cose necessarie all'uso e al sostegno della vita. ${ }^{82}$

Né mancava quindi di lodare la "fertilità della terra e dell'acqua", a cui faceva seguire la descrizione dei prodotti dell'agricoltura, dell'allevamento e della caccia, nonché dell'industria estrattiva. Botero conseguentemente veniva a esaltare l'organizzazione del lavoro che era stata instaurata al fine di provvedere al nutrimento di una popolazione così estesa:

A questa tanta fertilità della terra e dell'acqua s'aggiunge incredibile coltura dell'uno e dell'altro elemento, per la qual se ne cava tutto il possibile, il che procede da due cagioni: l'una è l'inestimabile moltitudine degli abitanti, perché si fa conto che la China faccia più di sessanta milioni d'anime; l'altra è l'estrema diligenza che si usa, e da' particolari in coltivare ed in cavar frutto da' loro poderi, e da' magistrati in far che a nessuno non sia lecito lo star ozioso e scioperato, sì che non vi è palmo di terreno che non sia benissimo coltivato. ${ }^{83}$

Venivano altresì registrate forme di lavoro coatto a cui erano sottoposti i disabili e le donne, infatti:

non è paese dove maggiormente [le arti] fiorischino per varietà e per eccellenza; il che procede da due cagioni: l'una si è quella [...] che ognuno si è sforzato a far qualche cosa, anco i ciechi, anco i monchi e gli stroppiati, se non sono affatto impotenti; e le donne, per una legge di Vitei, re della China, sono obligate a far l'arte del padre o almeno, per nobili e per grandi ch'elle si siano, ad attendere alla conocchia ed all'ago.

In verità, proprio la trasmissione dei segreti delle arti dai padri ai figli era assunta da Botero, anche con espressioni iperboliche, come ragione della perfezione e della grande varietà dei manufatti: "i figliuoli debbono necessariamente imparare il mestiere del padre, onde avviene

82 G. Botero, Delle cause della grandezza delle città, cit., 1. II, cap. XII, Della residenza del principe, p. 388.

83 Ivi, pp. 391-392. 
che gli artefici sono infiniti, e che i fanciulli appena nati sanno lavorare e le fanciulle istesse, e che l'arti si conducono a somma perfezione".

Da tali condizioni derivava una vera e proprio autarchia che sosteneva positivamente i traffici commerciali:

È tanta la copia finalmente e la varietà de' frutti della terra e dell'industria umana, che non hanno bisogno nessuno dell'altrui e danno grandissima quantità del suo a' paesi stranieri, e per non dir altro, la quantità della seta che si cava dalla China, non è credibile. ${ }^{84}$

Definiti in questo modo i fondamenti economici della potenza della Cina, l'attenzione che Botero rivolgeva alla Cina nella sua Ragion di Stato era, come sì è detto, intesa soprattutto all'analisi degli assetti politici che rendevano quella lontana "provincia" "ottimamente regolata", proponendola anzi come paradigma di razionalità per la costruzione di una efficiente burocrazia. Consigliando al principe "due diligenze, l'una nell'elezione, l'altra nella conservazione degli ufficiali" affinché, appunto, "si provveda di ministri [di giustizia] sofficienti e da bene", Botero lo invitava a "elezione di gente dotata, e di scienza, e di pratica necessaria" e a tal fine suggeriva proprio l'esempio dell'impero cinese:

Ma perché ho detto che si ricerca negli ufficiali pratica delle cose, non voglio lassar di dire che i re della China danno i magistrati per ordine, cioè a novizi i più bassi, e di mano in mano i più alti, accioché con l'isperienza di quelli si faccino scala a questi. ${ }^{85}$

Tale affermazione restava testimonianza peculiare di come la modernizzazione burocratica, che faticosamente si stava compiendo negli stati europei, potesse trovare un modello significativo in quella cultura dell'amministrazione che da secoli era praticata in Cina. Così, in un momento storico in cui in Europa dominava la venalità degli uffici,

84 Ivi, p. 392.

85 G. Botero, Della Ragion di Stato, cit., l. I, cap. XVI, De’ ministri di giustizia, p. 80. Si noti come Botero non facesse alcun rimando al sistema degli esami e facesse dipendere, anche per la Cina, la selezione dei magistrati dalla scelta del sovrano. Il capolavoro boteriano è stato riedito nel 2016 da Einaudi, a cura di Pierre Benedettini e Romain Descendre, con introduzione di Romain Descendre. 
Botero portava come esempio l'Impero di Mezzo per illustrare le procedure di controllo che il principe doveva attivare per garantirsi l'integrità dei magistrati:

il primo [modo] è di salariarli ed il vietar loro sotto pene gravissime il ricever presenti, il che fanno in modo singolare i re della China, perché provveggono i giudici e di viatico e di stanze, apparato, ministri, servitori e di tutto ciò che appartiene alla commodità e all'onorevolezza loro, sì che ad essi non resta altro pensiero, che d'attendere con tutto lo spirito all'amministrazione della giustizia e dell'ufficio commessoli. ${ }^{86}$

Vale notare, però, come non tutte le istituzioni e le pratiche di dominio dispiegate nel lontano impero incontrassero in Botero quella approvazione che, invece, avrebbero ricevuto nell'opera di Ricci. ${ }^{87}$ Infatti, se il gesuita maceratese, ricordando i "molti belli ordini del Governo" emanati da Hongwu, ne sottolineava il "grande ingegno e giudicio" - in primo luogo per decisione di allontanare dai centri di potere tutti i famigliari del sovrano ${ }^{88}$-, tutt'altra posizione era stata sostenuta sullo stesso tema da Botero. Proprio trattando delle sedizioni, e conseguentemente delle persone "la cui autorità e possanza può dare sospetto al prencipe" ${ }^{89}$ accomunava le azioni degli imperatori cinesi a quelle degli altri despoti orientali e africani, sottolineando il fatto che "né li re della China, né gli imperatori dell'Etiopia col confinar i parenti, né i Turchi con l'ammazzarli od i Mori con l'accecarli assicurano gli stati loro dalle sedizioni e da' sollevamenti".$^{90}$ Tuttavia, su un punto -

86 Ivi, 1. I, cap. XVII, Del contenere i magistrati in officio, pp. 83-84.

87 Nella consapevolezza che la pubblicazione della prima edizione sia della Ragion di Stato (1589) sia delle Relationi Universali (1590) di molto precede l'inizio della redazione del manoscritto di Della entrata della Compagnia di Giesù e della Christianità in Cina (1608), resta necessario osservare come il confronto più sopra instaurato tra Botero e Ricci non serva certo a evidenziare calchi citazionali ma, piuttosto, sia rivolto a sottolineare la pervasività di ben precisi quadri ideologici e valoriali, pur nella differente prospettiva che i due autori percorsero.

88 M. Ricci, Descrizione della Cina, cit., cap. VI, Del Governo della Cina, p. 66: "perché si vede chiaro nelle storie antiche che tutte le famiglie reali si persero o per ribellioni di parenti del Re o di altri stati particolari, per stare grande parte del Governo nelle loro mani", Hongwu stabilì "che nessun Parente del Re avesse mai nessun governo nel regno, né di Città né di soldati”.

89 G. Botero, Della Ragion di Stato, cit., 1. IV, cap. III, Dei Grandi, p. 160.

90 Ivi, 1. IV, cap. IV, De' prencipi di sangue, pp. 161-162, in cui esponeva 
centrale, perché metteva a confronto l'effettiva fungibilità di quelle che Botero considerava "parti" della Ragion di Stato ${ }^{91}$ - i due autori declinavano, pur con differenti prospettive teoriche, l'ideologia della conservazione, operando entrambi un decisivo confronto tra la politica di potenza condotta dai dinasti europei e la scelta tutt'affatto opposta a cui si erano attenuti gli imperatori orientali. ${ }^{92}$ Infatti, elencando le principali differenze che "per il buon governo" intercorrevano tra Cina ed Europa, Ricci avrebbe ricordato come i sovrani cinesi non coltivassero in alcun modo mire espansionistiche:

essendo questo regno sì grande e ripieno di gente e fornito di vettovaglia e materia per far legni, artigliaria et altri strumenti di guerra, con che potrebbero facilmente soggettar al loro dominio al manco tutti questi regni vicini, con tutto questo né gli Re né i sudditi si curano né trattano di questo, stanno contenti con il suo, senza volere quello degli altri. ${ }^{93}$

chiaramente le ragioni della sua critica: "Non i Chinesi e gli Etiopi, perché, quando ben i loro parenti siano d'animo quieto e ben composto, può essere che 'l popolo ed i baroni, concitati da sdegno o da furore, o mossi da paura di castigo o da desiderio di vendetta sollecitino i confinandi e, corrompendo o sforzando le guardie, gli cavino fuor delle prigioni e de' confini e li collochino in seggio". Inoltre tale pratica non garantiva in nessun modo la sicurezza del re: "Non nego però, che l'usanza de' Chinesi e degli Etiopi non abbino men del barbaro e dell'ingiusto, conciosiaché l'usanza ha forza di legge, ed è cosa ragionevole che, per liberare di pericolo o anche di sospetto il regno, i parenti del re si contentino di quel piacevole confine; ma non vi è però tutta quella sicurezza che si pensa, conciosiaché nella China sono stati ammazzati molti re e vi hanno dominato tiranni crudelissimi e sino alle donne".

91 Ivi, 1. I, cap. I, Che cosa sia Ragione di Stato, p. 55: "Stato è un dominio fermo sopra i popoli e Ragion di Stato è notizia di mezzi atti a fondare, conservare ed ampliare un dominio così fatto. Egli è vero che, sebbene assolutamente parlando ella si stende alle tre parti suddette, nondimeno pare che più strettamente abbracci la conservazione che l'altre, e dell'altre più l'ampliazione che la fondazione, imperocché la Ragion di Stato suppone il principe e lo Stato (quello come l'artefice, questo come materia), che non suppone, anzi, la fondazione affatto, l'ampliazione in parte, precede".

92 Sulla fine dell'espansionismo della dinastia Ming - e sul conseguente ripiegamento su una strategia solo difensiva adottata dalla Cina a seguito dell'ordine di rientro impartito a tutte le flotte nel 1421 dall'imperatore Zhu Di dopo l'incendio della Città Proibita - si veda G. Menzies, 1421. La Cina scopre l'America, Firenze, Carocci, 2003.

93 M. Ricci, Descizione della Cina, cit., cap. VI, Del Governo della Cina, p. 78. Per il vero, Diego de Pantoja nella sua Relatio dava altra e differente spiegazione a tale strategia. Infatti, oltre l'usuale motivazione della ricchezza di risorse di cui disponeva la Cina, affermava che "Neque Sinae neque Rex cogitationem ullam de promovendis 
Non a caso gli sarebbe corso facile il paragone con le pretese egemoniche e le guerre di conquista che avevano scandito continuamente la storia europea:

Certo assai diverso dalle nostre nationi, le quali soventemente perdono i proprij regni per volere signoreggiare agli altrui e che, per l'insatiabile voglia di allargare lo imperio, mai potero conservare il suo originale centinaia o migliaia di anni come fecero i Cinesi. ${ }^{94}$

Già affrontato nella Ragion di Stato, ${ }^{95}$ questo tema sarebbe ritornato ancora nelle Relationi universali in cui Botero non a caso presentava la potenza degli stati scandendone "ricchezze, governo, forze, prencipi confinanti", rielaborando un metodo che era stato seguito dagli ambasciatori di Venezia nelle loro relazioni al Senato della Serenissima, ed era iterato nelle numerosissime edizioni del Thesoro politico. ${ }^{96}$ Così, insieme alle risorse di cui poteva godere la Cina, "un paese poco minore dell'Europa", venivano descritte le prassi di governo seguite dai sovrani cinesi, assunte come paradigma di quella ideologia della conservazione che aveva connotato tutto il pensiero politico italiano tra Cinque e Seicento. Anche nelle Relationi Universali venne illustrata la "risolutione" presa dai sovrani cinesi di non voler più condurre guerre offensive, considerandole condizione controfattuale alla conservazione di quel "felicissimo paese":

regni terminis suscipiunt, quod adeo verum est, ut ne oblatas quidem sibi duas regiones sint accepturi". La ragione di questo comportamento risiedeva nel fatto che non si potessero poi trovare magistrati che andassero ad amministrare le terre conquistate: "Longeque vero aegrius, si longinque essent, quod cum caeteris, tum Mandarinis praecipue, ornatisque hominibus infortunatum esse videatur, e suo regno in alias oras exire", cfr. Iacobi Pantogia S. I. De amplissimo Sinarum regno relatio, cit., p. 250.

94 M. Ricci, Descizione della Cina, cit., cap. VI, Del Governo della Cina, p. 78.

95 G. Botero, Della Ragion di Stato, cit., 1. VII, cap. VIII, Dell'astenersi dalle spese impertinenti e dal dar vanamente, p. 235: "se non si ricerca tesoro infinito per la difesa, egli è molto meno necessario per l'offesa e per l'acquisto dell'altrui [...]. Molto maggior prudenza mostrano i Chinesi, perché quantunque essi signoreggiassino tutte quasi le isole dell'Oceano Eoo e la più parte dell'India, nondimeno, vedendo che un'impresa tale li consumava infinite ricchezze, armate, genti, sostanze, si risolsero di lasciarla e di ritirarsi nel loro paese facendo una legge per la quale si proibiva di navigare in quei paesi e di far la guerra offensiva".

96 A tali modelli faceva già riferimento F. Chabod, Appendici a "Giovanni Botero", cit., pp. 429-430. 
Li re si astengono affatto dalle guerre offensive; e in vero godendo essi di un felicissimo paese, ove contende la fertilità della terra con l'industria inestimabile de gli huomini; ove non manca cosa niuna e molte ne avanzano molto all'uso loro; a che fine consumar le loro facultà per far acquisti inutili allo stato? Logorar le forze e le sostanze proprie per acquistar l'altrui? ${ }^{97}$

Consapevole che tale scelta veniva a contrastare con le logiche espansionistiche perseguite dalle potenze europee, Botero ne sottolineava anzi il valore davvero paradigmatico, distinguendo nettamente la prudenza, che informava l'azione degli europei, dalla sapienza testimoniata dai cinesi:

Questa risolutione de' Chinesi, se ben non sarebbe approvata da la prudenza moderna, si deve però stimare piena di saviezza. Perché non è sciochezza maggiore, che il perdere il suo per far acquisto dell'altrui; il dissipar forze per allargar i confini $[\ldots]$ : molto maggior opera d'un Prencipe è il conservare (come abbiamo dimostrato altrove) che l'aggrandire.

Vale notare, tuttavia, che nella Ragion di Stato e nelle Relationi Universali la strategia difensiva perseguita dai sovrani cinesi veniva ascritta a due ben differenti campi semantici: se nella Ragion di Stato tale scelta ricadeva nei dispositivi prudenziali illustrati nei capitoli I e VI del secondo libro, nelle Relationi Universali era invece testimonianza di "saviezza”, ovvero di una guida razionale dell'azione politica, che, proprio per questo, non si esauriva nei tatticismi congiunturali dettati dalla prudenza. E se nelle Relationi Universali ritornava - di necessità, a motivo dell'impianto stesso dell'opera - la descrizione di tutte quelle caratteristiche che facevano della Cina un "regno non meno ampio, e

97 G. Botero, La seconda parte delle Relationi Universali [...]. Nella quale si discorre della potenza de' maggior Prencipi, che siano al mondo: E le cagioni della grandezza, e sicurezza de' loro stati, In Brescia, Appresso la Compagnia Bresciana, 1599, p. 91. Per le citazioni dell'opera ci si è avvalsi di tale edizione perché considerata dallo stesso Botero la migliore tra tutte quelle che si erano succedute fino al 1600, si veda la Lettera dell'autore sopra le sue Relationi al Signor Don Antonio Lopez di Calataiut, apparsa in G. Botero, I Prencipi [...] con le aggiunte alla Ragion di Stato nuovamente poste in luce, Torino 1601, pp. 148-149. Nel 2015, presso l'editore Aragno di Torino, sono apparse le Relazioni Universali, a cura di Blythe Alice Raviola, in due volumi. 
spacioso, che raccolto, e unito, e pieno di gente, vettovaglie, e di ricchezze $[\ldots]$ uno de' grandi imperii, che sia mai stato", ${ }^{8}$ l'analisi del governo riprendeva ed espandeva con accuratezza la traccia contenuta nella Ragion di Stato, ascrivendola, però, alla forma dispotica:

Il governo della China ha del dispotico ${ }^{99}$ assai: conciosiache non è in tutta la China altro signore, che il Re; né sanno che cosa sia Conte, Marchese, o Duca; né vi è altro a cui si paghi tributo o gabella. Il Re conferisce tutti i Magistrati, e la nobiltà istessa. Egli somministra loro il modo di mantenersi; e non fanno cosa alcuna d'importanza, della quale non ne sia avvisato. Onde egli non è solamente ubidito come un Re, ma quasi adorato come Dio. ${ }^{100}$

Conseguente alla divinizzazione della figura del sovrano la pagina delle Relazioni Universali testimoniava il più completo asservimento dei sudditi: "e non solamente il Re, ma i Presidenti ancora, e i Giudici sono riveriti di tal sorte, che non si parla loro se non in ginocchio: il che avvilisce grandemente gli animi de' popoli, e li rende schiavi anzi, che sudditi del lor Prencipe". ${ }^{101}$

Per il vero, non era questa l'unica occorrenza che il termine dispotico incontrava nelle pagine della Seconda parte delle Relationi Universali; non a caso, con la formula "dispotico assai", compariva anche a descrivere il governo del Prete Gianni:

Il governo del Prete Gianni ha del dispotico assai, perché tiene i suoi vassalli bassissimi, non meno i grandi che i piccoli; e li tratta più presto come schiavi, che come sudditi, e per far ciò meglio, si mantiene presso loro quasi in riputatione, di cosa sacra e divina. ${ }^{102}$

Tutt'altro che frequente nella letteratura politica d'età moderna,

98 G. Botero, La seconda parte delle Relationi Universali [...]., cit., p. 96.

99 Sulle varie caratterizzazioni che il concetto di dispotismo ebbe nel pensiero politico occidentale, da Aristotele a Voltaire, pionieristico resta il saggio di R. Koebner, Despot and Despotism: Vicissitudes of a Political Term, Journal of the Warburg and Courtauld Institutes, 14, 3/4, 1951, 275-302, e ancora importantissimo è il volume di Karl August Wittfogel, Il dispotismo orientale, Milano, Sugarco, 1980.

100 G. Botero, La seconda parte delle Relationi Universali [...]., cit., p. 96.

101 Ibidem.

102 Ivi, p. 153. 
come osservava Koebner, il termine 'dispotico' in Botero veniva a connotare non solo quella dimensione servile propria, secondo Aristotele, ${ }^{103}$ a popoli barbari soggetti a un governo arbitrario nei comportamenti del monarca e, tuttavia, ereditario conforme alla legge, ma anche un governo che era espressione della divinizzazione della figura del sovrano o - come avrebbe scritto Montesquieu - "la cui natura è di riunire tutti i poteri in una sola persona". ${ }^{104}$ Tuttavia, il giudizio formulato da Botero sembrava far della Cina un modello perfettamente consolidato grazie a quelle prassi conservative della comunità politica che era stato scopo precipuo della sua Ragion di Stato prescrivere:

è finalmente regno regolato di tal maniera, che non ha altra mira che la pace, ${ }^{105}$ e la conservazione dello stato: e per questo vi fiorisce la giustitia, madre della quiete, e la politica maestra delle leggi, e l'industria figluola della pace: e non è regno né dominio antico, né moderno meglio regolato di questo. Conciosia che sono già più di due milla anni (per quanto essi dimostrano nell'historie loro) che si governa con le

103 Aristotele, Politica, III, 8, 1279b; III, 14, 1285a; IV, 10, 1295a. Con nettezza Tolomeo da Lucca, nel portare a termine il De regime principum di Tommaso d'Aquino, avrebbe definito (1. II, 9) il governo dispotico in questo modo: "Est autem hic advertendum quod principatus despoticus dicitur qui est domini ad servum", cfr. R. Koebner, Despot and Despotism: Vicissitudes of a Political Term, cit., p. 279.

104 Ch. de Secondat barone di Montesquieu, Lo spirito delle leggi, cit., vol. II, 1. XXV, cap. VIII, p. 131.

105 Che il mantenimento della pace fosse costante obbiettivo della politica imperiale cinese trova una conferma, tra le altre, in una pagina di M. Martini, Breve historia delle guerre seguite in questi ultimi anni tra Tartari e Cinesi. Nella quale si racconta come $i$ Tartari in sette anni hanno occupato quasi tutto il vastissimo Imperio della Cina. E si dà breve contezza de' costumi de' medesimi Tartari. Scritta in latino dal R. P. Martino Matini Trentino [...] Et tradotta in Italiano dal Sig. Climaco Latini, In Milano, per gli Heredi di Gio: Battista Bidelli, 1654, p. 8: pronto a concludere la pace con i Tartari occidentali, l'imperatore Nungijo "ogni anno mandava loro tributi sotto coperta di donativi. Né ciò si stima disdicevole fra Cinesi per ripararsi dalla guerra, la quale per detto de' loro Filosofi è molto dishonorata cosa, e da valersene solo, quando tutti gli altri mezzi alla conservazione, e quiete delle provincie venissero meno", senza trascurare, tuttavia, di adottare dispositivi militari volti a dissuadere il nemico dal portare attacchi: "[i Cinesi] non lasciarono mai le frontiere della loro Monarchia senza grossi presidij, sì che per tenere ben guardato quel lunghissimo muro [la Grande Muraglia] vi mantenevano un millione di soldati". Quest'opera, come è noto, fu "the first detailed information about the Manchou conquest of the China", cfr. D.F. Lach e E.J. van Kley, Asia in the making of Europe. Volume III. A century of advance. Book one: trade, missions, litterature, cit., p. 525. 
medesime leggi; e pure noi ci meravigliamo della Republica di Venetia, che s'è mantenuta 1100 anni, e del Regno di Francia, che s'è conservato $1200 .^{106}$

Vale osservare che proprio queste ultime due citazioni, per altro tratte dal medesimo capitolo della Seconda parte delle Relationi Universali (Re della China. Governo), danno conto in modo puntuale di come la descrizione della Cina possa differentemente articolare la riflessione politica nell'Italia tra XVI e XVII secolo. Il primo brano mostra come le categorie politiche elaborate dal pensiero occidentale fossero rese funzionali a una precisa inclusione della realtà cinese nelle consuete tassonomie delle forme di governo. Il secondo brano è inteso, invece, a confermare il valore fondativo del principio di conservazione della comunità politica, riconoscendo anzi all'impero orientale un primato di durata e, conseguentemente, di perfezione, tema che era stato oggetto di lunga disputa nella letteratura politica della prima età moderna. In meno parole: l'impero cinese non era solo una realtà che poteva essere

106 G. Botero, La seconda parte delle Relationi Universali [...]., cit., p. 97. Per il rapporto complesso che nel pensiero politico italiano di Cinque e Seicento le tradizioni filomonarchica e filorepubblicana seppero costruire tra l'ideologia della conservazione e l'esaltazione della perfetta organizzazione della comunità politica (in meno parole: durata di un governo versus la sua perfezione) si veda P.C Pissavino, Le ragioni della Repubblica. La "Città felice" di Lodovico Zuccolo, San Marino, Centro Sammarinese di Studi Storici, Università degli Studi della Repubblica di San Marino - Aiep, 2007, pp. 199-223. In verità, Bodin dava una spiegazione ben differente alla lunga durata che aveva caratterizzato la storia delle monarchie dispotiche: "La ragione per cui la monarchia dispotica è più durevole delle altre [forme di monarchia] sta nel fatto che è più augusta, e che i sudditi dipendono interamente, quanto a vita, libertà e proprietà, dal sovrano che li ha conquistati a giusto titolo, cosa che abbatte completamente ogni loro ardire, così come lo schiavo, conscio della sua condizione, diviene generalmente umile, vile, di animo servile insomma, come appunto si dice", cfr. J. Bodin, I sei libri dello Stato, cit., vol. I, 1. II, cap. II, Della monarchia dispotica, p. 579. Varrà ricordare che la prima traduzione italiana, redatta dal genovese Lorenzo Conti nel 1588, definiva tale forma di monarchia come "Monarchia Signorile", ovvero quella in cui "il Principe è fatto Signore de beni, e delle persone per ragioni d'arme, e di buona [giusta] guerra, governando i sudditi, come il padre di famiglia fa i suoi schiavi”, cfr. I sei libri della Republica del Signor Giovanni Bodino [...], cit., p. 167. Per completezza si segnalano anche le seguenti varianti di traduzione del brano più sopra riportato: "più augusta" (trad. 1964) era stato reso da Conti "ha un non so che di maggior maestà in sé", e "il sovrano che li ha conquistati" era stato invece qualificato come "Principe assoluto": entrambe le citazioni si leggono nella traduzione del 1588 a p. 170. 
compresa, pur nella sua alterità, solo applicandovi una strumentazione teorica che sin dall'antichità era stata attagliata all'esperienza politica e alle prospettive ideologiche europee. Con ogni evidenza, nella scrittura di Botero la Cina diventava così la conferma storica e anzi la realizzazione perfetta del modello politico che la tradizione della dottrina della ragion di stato veniva a prescrivere.

\section{LA CinA In LODOVICO ZuCCOlO: TRA “FOGGIA Sì ESQUISITA DI GOVERNO” E MODELLO PER UTOPIA}

Se in Botero la Cina appariva come modello delle pratiche di conservazione dello stato, in Lodovico Zuccolo divenne chiaro esempio di come alcuni caratteri del suo sistema sociale e politico potessero essere assunti come elementi costitutivi dell'ottimo stato, se non addirittura della costruzione utopica. Vediamo come.

Per Zuccolo la stabilizzazione/perfezione di una comunità politica non doveva basarsi solo su dispositivi prudenziali che potevano dispiegarsi come prassi repressive ma doveva articolare anche forme di giustizia premiale. Infatti, di fronte alla corruzione presente nella società, Zuccolo sosteneva l'importanza dell'onore e della vergogna nel mantenere il popolo in pace e in quiete:

Né perché gli animi sì infermi come hora si trovano, non si possono medicare se non col castigo, resta però, che quando si attendesse da dovero a levare via le cagioni delle infirmità, non si potessero ridurre a sì buona composizione, che poco più facesse di mestiero la pena, ma più riuscissero opportuni l'Honore, e la vergogna.

A darne esempio, lo Zuccolo richiamava - accanto ai grandi modelli politici e civili dell'antichità classica: Sparta e a Roma - anche la Cina:

Non ho ben contezza di che costumi fossero i popoli della China, prima che vi fosse introdotta la civiltà presente: ma voglio bene credere, che havessero del barbaro, e del fiero. Nondimeno il senno, e la prudenza di quel buon Filosofo, il quale diede loro la maniera del vivere, furono di sì rara esquisitezza, che (se le relationi, le quali habbiamo di colà, non sono del tutto false) si regge un Imperio immenso, e potentissimo già per lo spazio di due migliara d'anni con tanta giustitia, \& equità, che premio, pena, honore, vergogna sono con sì ben intesa pro- 
portione distribuiti, che né merito, né demerito si possono dare senza giusto compenso di bene, o di male: foggia sì esquisita di Governo, che forse non fu mai veduta altrove. ${ }^{107}$

La Cina diveniva così un approdo di quella ricerca sulle "ragioni del governo" che costituirono l'oggetto pressoché costante dell'indagine Zuccolo, ${ }^{108}$ in cui all'analisi empirica dei fenomeni politici seguiva la costante riflessione sull'ottima forma di governo, delineata anche nella sua utopia, La Repubblica d'Evandria. Invero, il fatto che Zuccolo avesse descritto, per primo nel dottrinarismo italiano, la "convenienza" che doveva intercorrere tra ragion di stato e ogni specifica forma di governo apriva la sua analisi a un relativismo ${ }^{109}$ che conseguentemente riduceva l'alterità che caratterizzava la Cina, avanzando così le specifiche ragioni che giustificavano l'assunzione di suoi peculiari assetti sociali a segmen-

107 L. Zuccolo, Discorso dell'Honore, in Id., Discorsi dell'Honore, della Gloria, della Riputazione, del Buon Concetto, In Venetia, Presso Marco Ginami, 1623, pp. 155. 156. Vale ricordare che sulle prassi premiali che scandivano l'amministrazione della giustizia in Cina si era soffermato anche Michel de Montaigne nei suoi Essais: "gli ufficiali incaricati dal principe di ispezionare la condizione delle sue province, come puniscono quelli che commettono concussione nella loro carica, così compensano con pura liberalità quelli che vi si sono condotti bene, al di là del modo comune, e al di là della necessità del loro dovere. Ci si presenta loro non solo per giustificarsi, ma per acquistarvi e non semplicemente per essere pagati, ma anche per essere gratificati", si veda $M$. de Montaigne, Saggi, a cura di F. Garavini, con un saggio di S. Solmi, Milano, Adelphi, 1992, la citazione è tratta dal l. III, cap. XIII, Dell'esperienza, vol. II, p. 1433. Per la diffusione dei Saggi di Montaigne nella cultura italiana si veda G. Cozzi, Venezia barocca. Conflitti di uomini e di idee nella crisi del Seicento veneziano, Venezia, Il Cardo, 1995. Vale ricordare che l'opera di Montaigne, Saggi [...] Overo discorsi naturali, politici e morali, apparve per la prima volta "In Venetia, Presso Marco Ginammi, 1633".

108 Che tale tema fosse il centro della speculazione di Zuccolo trova conferma nell'avviso L'Auttore a chi legge premesso alla seconda edizione delle sue Considerationi politiche, e morali sopra cento oracoli d'illustri personaggi antichi. [...] che il frontespizio recita come Rivedute, e corrette, et aggiuntavi una breve risposta alle Oppositioni dell'Accademico Pellegrino, In Venetia, Appresso Marco Ginami, 1623. Vale ricordare che la prima edizione dell'opera rimonta al 1621, sempre presso il medesimo editore.

109 Ivi, Oracolo XI, Della Ragion di Stato, pp. 54-73: per Zuccolo, infatti, la "ragion di stato tutta si rivolge intorno al conoscere que' mezzi e avvalersene, i quali siano opportuni per ordinare o per conservare qualsivoglia costituzione di republica, qualunque ella si sia", si legga l'Oracolo in B. Croce e S. Caramella (a cura di), Politici e moralisti del Seicento. Strada-Zuccolo-Settala-Accetto-Brignole Sale-Malvezzi, Bari, Laterza, 1930, pp. 27-28. 
ti costitutivi di un ordine politico descritto come perfetto. Infatti, nella repubblica d'Evandria la Cina, a partire dagli stessi materiali citazionali usati da Botero, venne addirittura a figurare alcune delle condizioni di esemplarità utopica. In Evandria le arti "passano, come hereditarie, da padre a figliuolo. Talché le arti si riducono, come pure scrivono de' Cinesi e si legge altresì degli antichi Egizi, a esquisita finezza”. Accanto a queste citazioni, si possono scorgere altri rimandi, che facevano riferimento, con la prescrizione dell'isolamento, a una condizione fondamentale dello statuto di perfezione politica proprio del genere utopico. Così in Evandria "anco i ciechi, i zoppi, gli stroppiati s'impegnano in qualche esercizio", e alle frontiere le guardie controllavano "che non v'entrino altri, che ambasciatori e mercanti, o qualche gentiluomo, o personaggio honorato", mentre "non si concede agli huomini di Evandria a partire a voglia loro dalla Provincia". ${ }^{110}$ Una glossa a margine avvisava inoltre, che gli Evandri imitavano i cinesi nel rifiutare ogni aumento territoriale: "Ne manco i cinesi vogliono fare nuovi acquisti". Sicché, alcuni segmenti del sistema descrittivo elaborato dalla tradizione memorialistica gesuitica e di viaggio, volti a illustrare il modello sociale e politico cinese, erano adattati a architettura compositiva della città ideale. Così, anche in Zuccolo veniva meno quella radicale polarizzazione tra realtà politiche europea e modi di governo praticati in Cina.

\section{L'IDEOLOGIA DELL'ORDINE: IL GOVERNO DELLA LEGGE E LA MECCANICA DEL COMANDO}

Il gesuita Daniello Bartoli, per propria parte, volle ricompilare le aduse descrizioni dell'autocrazia esercitata dal sovrano cinese in una riflessione su quel regime politico che, da un lato, acquisiva nuove prospettive grazie agli studi scientifici di cui era cultore per nulla saltuario, ${ }^{111}$ dall'altro, ancora, si avvaleva di altri fondamentali concetti politici

110 Id., Il Porto, overo Della Republica d'Evandria, in Id., Dialoghi, cit., pp. 219, 226, $215,228$.

111 Sugli interessi scientifici coltivati da Bartoli e, in generale, sulla sua attività letteraria si vedano A. Asor-Rosa, Bartoli, Daniello, in Dizionario Biografico degli Italiani, vol. VI, Roma, Istituto della Enciclopedia Italiana, 1964, pp. 563-571, e il rimando alla sua opera contenuto in D.F. Lach e E.J. van Kley, Asia in the making of Europe. Volume III. A century of advance. Book one: trade, missions, litterature, pp. 381-382. 
che andavano oltre alla mera ideologia della conservazione ribadita dalle scritture utopiche e sulla ragion di stato. Vediamo come.

Pur convenendo che il "Re della Cina" fosse un "principe d'assolutissimo imperio", ${ }^{112}$ il gesuita esaltava il "maraviglioso provvedimento" che poneva freni all'arbitrio regio. Infatti, consapevoli che il regime monocratico potesse sempre degenerare in tirannide, "gli antichi savi della Cina" stabilirono che il re dovesse attenersi scrupolosamente a quanto gli era indicato dai consiglieri. ${ }^{113}$ Tale risultato era stato ottenuto "obligando il Re a non voler altro che il giusto, e 'l retto: e giustissimi, e rettissimi sono, né possono essere altramente i suoi ministri [...]: onde per conseguente, egli de' volere ciò che essi consigliano". Attraverso tale prassi, avvertiva Bartoli, non avveniva però né cessione né trasferimento di sovranità, e neppure si stabiliva quella forma mista di governo che pure era parsa a Ricci costituire la profonda realtà della politica cinese:

in ciò punto si deroga a quella sovranità, e signoria, che non ammette soggettione, o scemamento di potestà in un Monarca, conciosia che il poter volere ciò che è ingiusto, e operare ciò che è iniquo, non è potestà che si richieggia in un Principe, anzi che non sia meglio il mancarne, che haverla.

Che, poi, una efficiente burocrazia fortemente gerarchizzata fosse condizione "per l'eccellenza del [...] Governo" della Cina era riflessione che venne puntulmente testimoniata da Giovan Francesco Gemelli Careri (1651-1725) nel suo Giro del Mondo. Il Careri ricordava infatti che

Fra le tre leggi che son quivi tenute, quella de' Letterati è la prima, e la più antica; il suo fin principale è il buon governo del Reame: su la

112 D. Bartoli, Dell'Historia della Compagnia di Giesù. La Cina terza parte dell'Asia [...], In Roma, nella stamperia del Varese, 1663, p. 98, se ne veda ora l'edizione a cura di Bice Mortara Garavelli, Bompiani, Milano 1975.

113 Ibidem: "Ma nondimeno, percioché si doveva eleggere l'ottimo della Monarchia, con tale avvedimento, che non havesse a temersene il pessimo della Tirannia, dove avvenisse, che il Re, o per potenza assoluta da ogni timore, o per giovanile baldanza, dispregiati, o non attesi i buoni consigli, maneggiasse il governo a capriccio di libertà, non a regole di dovere, maraviglioso è quel provvedimento che si trovò da quegli antichi savi, a far che i consigli senza punto uscir de' loro termini si eseguiscano come fosser comandi”. 
quale eglino han composto gran numero di libri, e di commentarj. Confucio fè ne' suoi tempi un Trattato sopra tal soggetto che intitolò Chum yum, cioè a dire la Mediocrità dorata [...].

Dopo l'omaggio a Confucio, Careri descriveva puntualmente l'organizzazione gerarchica dei mandarini che "in tutto l'impero si distinguono in nove ordini: ed ogni ordine è diviso in nove gradi" e si soffermava poi a sottolinearne la stretta gerarchizzazione:

La conoscenza, la distinzione, e la subordinazione di questi ordini è sì perfetta, la sottomessione, e venerazione degli ultimi verso i primi, e l'autorità di quelli sopra questi è sì grande, ed in fine la potenza sovrana del Re sovra gli uni, e gli altri è sì assoluta, che non è comparabile al nostro governo, o che sia Secolare, o Ecclesiastico. ${ }^{114}$

In verità, ancora prima di Careri, Bartoli aveva posto l'accento sul governo della legge come norma indefettibile per garantire la perfetta realizzazione dell'ordine politico: "Hor la rettitudine, così de Ministri nel consigliare, come del Re nello statuire, tutta è presa dalla norma inflessibile delle leggi". ${ }^{15}$ Quindi, a impedire le degenerazioni in cui poteva incorrere, il monarca doveva continuamente adeguare la propria volontà al rispetto della legge: "Il Re, col veramente poter tutto, e fare il tutto egli solo $[\ldots]$ non però si ardirà a voler fare cosa niuna diversamente da quel che le leggi dispongono, e l'uso antico prescrive". ${ }^{116} \mathrm{La}$ solitudine e la separatezza del monarca trovava, insomma, conforto nelle leggi e nel consiglio dei savi, ma soprattutto nel principio d'ordine che pareva introdurre una visione meccanicistica della politica. Bartoli chiedendosi, infatti, come

114 G.F. Gemelli Careri, Giro del Mondo [...]. Parte quarta contenente le cose più ragguardevoli vedute nella Cina, In Napoli, Nella Stamperia di Giuseppe Roselli, 1700. Notizie su questo funzionario togato dell'amministrazione del Vice Regno di Napoli, che a causa delle persecuzioni subite da un potente personaggio, compì lunghissime peregrinazioni in Europa e nel mondo si vedano il volume Viaggiatori del Seicento, cit., pp. 683-684 e D.F. Lach e E.J. van Kley, Asia in the making of Europe. Volume III. A century of advance. Book one: trade, missions, litterature, pp. 386-387. Importante è la voce reatta da P. Doria, Gemelli Careri, Giovanni Francesco, in Dizionario Biografico degli Italiani, vol. LIII, Roma, Istituto della Enciclopedia Italiana, 2000, pp. 42-45.

115 D. Bartoli, Dell'Historia della Compagnia di Giesù. La Cina terza parte dell'Asia [...], cit. p. 98.

116 Ibidem. 
essere possa, anzi pur come sia, che un Re, che nulla vede, tutto risappia, e tutto operi; e concorrendo innanzi a lui gli affari d'una sì gran monarchia, questi né si confondono con la diversità, né si opprimano con la calca, né i grandi tolgano l'udienza e la spedizione a' piccoli,

ne illustrava la "cagione": "la quale come che veramente sia un congiunto di molte insieme, nondimeno quella che tutte le muove, e le applica al ben operare, è l'Ordine", del quale, appunto, sono custodi i "Letterati". Infatti, se "il publico reggimento non si commette che alla sola classe de' Letterati”, la loro funzione conseguentemente diveniva "ordinare alla perfettione, al mantenimento, alla pratica del buon governo ciò che studiano, e ciò che insegnano; né in altro maggiormente faticano, che nella conservatione dell'Ordine". In questo modo "lo studio di que' savi” era stato sempre rivolto a portare a efficacia ed efficienza l'amministrazione dello stato, rendendo evidenti e certi i rapporti gerarchici tra le magistrature e le funzioni di governo. Il risultato di tale impegno era stata la costruzione di "una sempre durevole macchina di governo, che muovendo le cose di un sì vasto imperio, nondimeno riesce al maneggiarla sì facile, che più non si farebbe una ben regolata famiglia". Non a caso "Tutto è così ben compartito per ordine, e concatenato per soggettione e dipendenza de' gradi l'un superiore all'altro, che nol può esser meglio qualunque machina d'ingegnere, composta di molte ruote, che si vadano communicando l'un l'altra il moto". ${ }^{117}$

117 Ivi, pp. 87-88. Come si vede in Bartoli emerge un'immagine originale, meccanicistica, dello stato e della sovranità che non si ricollega né alla concezione organicistica di ascendenza aristotelica né a quella di persona civilis o ficta che Hobbes aveva elaborato nel De cive (cap. V, 9-12), nel Leviathan (cap. XVI) e nel De bomine (cap. XV). Nel pensiero politico italiano del Seicento Zuccolo ricorre a strumenti meccanici per costruire un'efficace metafora della politica, quando, indagando Quale sia l'ottima tra le forme di Governo - come recita l'oracolo XII delle sue Considerationi politiche, e morali -, poneva a confronto repubblica e regno: "Meglio si stabilisce, et si conserva il Regno che non fanno le Republiche, per haver bisogno di manco mezi a conseguire il suo fine". La prospettiva aristotelica chiaramente seguita da Zuccolo, qualificando la finalità della politica come perfezione nella distribuzione della virtù tra i cittadini e non nel solo re, permetteva di instaurare una metafora tratta dal mondo delle tecniche: "Con più facilità faremo una tenaglia, od una lima, che non un horologio da ruote, per haver bisogno questo di più mezi a far l'operation sua, che non ha una lima, o la tenaglia, et così anco di maggior diligenza di ben operare; ma sarà più nobile il fin dell'horologio, che quello della tanaglia, o della lima". Che, poi, l'attenzione di Zuccolo fosse 
Resta ovvio che lo schema meccanicistico di questa monarchia amministrativa superava, per la logica dell'efficienza ed efficacia dell'azione di governo, l'immagine tutta platonizzante che Athanasius Kircher e Martino Martini avevano elaborato per descrivere il ruolo che i letterati/ filosofi ricoprivano nel sistema politico cinese. Lontano, dunque, Bartoli dal risolvere la sua riflessione in una semplice ripetizione del governo dei filosofi-re, rimodulava la teorizzazione platonica in una innovativa immagine della politica, che, al tempo stesso, aggiornava la cultura dei dispositivi di conservazione della comunità civile prescritti dalla ragion di stato nell'ideologia dell'ordine meccanicistico del governo. D'altronde, a sancire l'ineluttabile deriva della concezione prudenziale della politica, il segretario della accademia fiorentina del Cimento, Lorenzo Magalotti (1635-1712), avrebbe sostenuto - di fronte alla conquista della Cina ad opera dei Manciù nel 1644 - che la dottrina di Machiavelli non era più "tale arcano di sottigliezza politica, che non v'arrivino ancora le grossolane menti dei Tartari”. ${ }^{118}$

rivolta a esaltare l'eccellenza della repubblica piuttosto che l'efficacia e l'efficienza ottenuta nell'arte del comando dal regno è esito, per così dire, scontato: "più pronto e più spedito nelle operationi sue hassi a stimare il Regno, che la Republica. Ma il fine della Republica dee bene di gran lunga preporsi a quello del Regno. Poiché nella Republica, massimamente in quella degli Ottimati, si mira ad introdurre virtù eminenti regie in tutte i cittadini", cfr. L. Zuccolo, Considerationi politiche, e morali sopra cento Oracoli d'Illustri personaggi antichi [...], cit., p. 75-76. Per l'orologio come metafora della complessità, specie in campo politico, si veda il saggio di O. Mayr, La bilancia e l'orologio. Libertà e autorità nel pensiero politico dell'Europa moderna, il Mulino, Bologna 1988.

118 L. Magalotti, Relazione della China cavata da un ragionamento tenuto col $P$. Giovanni Grueber della Compagnia di Gesù nel suo passaggio per Firenze l'Anno 1665, in Id., Varie operette [...] ora per la prima volta raccolte e non più stampate in Venezia, Venezia, Appresso Pietro Pizzolato e Compagni, 1779, pp. 99-100: "Il Re presente è un fanciullo di dodici anni in tredici; e che risiede a Pequin Metropoli del Regno, e così aver fatto il Padre, e l'Avo. Tanto, che il precetto di Machiavello, che per assicurarsi un Principe nuovo in uno stato nuovamente acquistato, disforme di lingue, di costumi, e di leggi dell'antico suo dominio, non vi è più sicuro partito, che l'andarvi ad abitare, non è tale arcano di sottigliezza politica, che non vi arrivino le grossolane menti de' Tartari”. Va detto che la prima edizione delle Varie operette risale, però, al 1697. Per il rimando al Segretario Fiorentino, si veda N. Machiavelli, Il Principe, a cura di M. Martelli, corredo filologico a cura di N. Marcelli, Roma, Salerno, 2006, cap. V, Quomodo administrandae sunt civitates vel principatus, qui antequam occuparentur suis legibus vivebant, p. 107: "Quando quelli stati che si acquistano come è ditto sono consueti a vivere con le loro leggi e in libertà, a volerli tenere ci sono tre modi: el primo ruinarle; l'altro, andarvi a abitare personalmente; il terzo lasciarle vivere con le sua leggi". 
Sicché all'esaurimento del primato della prudenza nel campo della politica, consapevolmente testimoniato dai maggiori pensatori dell'età della Controriforma, ${ }^{119}$ Bartoli sapeva contrapporre il modello di monarchia assoluta cinese incentrato sulla necessità di prassi burocratiche ben rodate sottoposte al dominio della legge, cassando, inoltre, ogni istanza alla formula della costituzione mista.

\section{PaOlo MatTIA Doria: IL PARADOSSO DELL'IMPERO CINESE}

Se la descrizione del sistema politico cinese in Bartoli si appoggiava a un seppur elementare paradigma meccanicistico, tuttavia all'inizio del Settecento non sarebbe mancata penna a restaurare il primato della interpretazione filosofica, confuciana dunque, della superiorità della Cina, additando, anzi il paradosso che aveva scandito, negli autori fin qui incontrati, la descrizione del suo assetto politico.

Modello di un sistema politico in cui "la filosofia è sempre quel fonte, dal quale scaturiscono le norme per coltivare i popoli nella civiltà", ${ }^{120}$ dell'impero di Cina Paolo Mattia Doria (1667-1746) propo-

119 Si veda, ad esempio, come il conte Virgilio Malvezzi (1599-1654), uno dei maggiori pensatori politici italiani del Seicento, portasse un durissimo attacco alle pretese avanzate dal dottrinarismo del suo tempo: “È un'arte da perso la politica: non v'è chi fino a ora l'abbia imparata. Molti sanno fare quello che s'avrebbe da fare: nessuno quello che s'ha da fare. Chi non conosce i movimenti della fortuna, non è buon politico. Chi li conoscesse conoscerebbe Iddio. Non si vede in faccia questi, ma solo per le spalle; perché del suo operare sono per dir essere ignoto tutto ciò che non è passato". Infatti, in questa pagina era dichiarato uno scacco gnoseologico a segnare l'esaurimento della politica prudente, e di tutta la tradizione filosofica che aveva discettato di politica avendo a fondamento tale concetto: "Il non servirsi della prudenza è bestialità: il fidarsene arroganza. L'uomo per operar bene, quantunque pieno di scienza, ha bisogno di un non so che di più, che non si può né apprendere né insegnare, né conoscere d'aver, se l'esperienza non glielo mostra, né che cosa si sia dopo averlo sperimentato", cfr. V. Malvezzi, L'Alcibiade, in Id., Considerationi con l'occasione d'alcuni luoghi delle vite d'Alcibiade e di Coriolano, In Bologna, per gli eredi del Dozza, 1648, si cita da B. Croce e S. Caramella (a cura di), Politici e moralisti del Seicento [...], cit.., pp. 264-265.

120 P.M. Doria, Il politico alla moda di mente adequata e prattico. Lettera nella quale si fanno alcune considerazioni intorno al Ministerio del sig. Cardinale di Fleury, $\mathbb{S}$ Dell'Imperio della Cina e della sua filosofia politica, in V. Conti, Paolo Mattia Doria. Dalla Repubblica dei Togati alla Repubblica dei Notabili, Firenze, Olschki, 1978, p. 231. Per ulteriori indicazioni bio-bibliografiche si veda almeno P. Rovito, Doria, Paolo 
neva nel suo Politico alla moda un ritratto complesso ed elogiativo, presentato a contraltare del degrado in cui il "dispotismo", praticato dai principi europei, era "la cagione della miseria, che soffrono i popoli". Ancora una volta, l'impero cinese diveniva in Doria termine di confronto con l'assetto politico vigente in un Europa, nei cui stati, per sua ammissione,

quella filosofia dalla quale si deduce vera morale, e vera politica, è in tutto sbandita [...] dai gabinetti de' principi assoluti, e dai consigli di quegli stati, che professano di governarsi con leggi di libertà. [...]. Negli stati governati con la sola assoluta libertà del Principe, li principi hanno autorità di eleggere per ministri di politica uomini ignoranti. Or questi sono quasi sempre vili adulatori delle passioni del Principe, onde poi sacrificano la libertà del popolo all'avidità di dominio, la quale sempre arde nel core de' principi. ${ }^{121}$

In verità, per Doria, "la scienza dalla quale si deduce il buon governo dello stato è la sola metafisica unita alla buona religione", ${ }^{122}$ così che "la lunga durata, che ha avuto l'imperio della Cina, la felicità che hanno goduto nel loro governo li Cinesi, non può essere stata cagionata da altro, che dalla buona disciplina, o sia filosofia, colla quale è stato governato quell'imperio". ${ }^{123}$ Non a caso, infatti, Doria riteneva che la filosofia di Confucio fosse stata "in tutto uniforme a quella di Platone, a quella di Pitagora, e a quella degli altri filosofi metodici, e metafisici" e, conseguentemente, restasse "la cagione del prudente e felice governo che hanno praticato i Cinesi". ${ }^{124}$ Vediamo, quindi, come Doria avesse descritto l'assetto politico della Cina.

Per il vero, per Doria l'imperatore della Cina è "dispote", e, "come sono il re di Persia, il Gran Soltano de' Turchi, assoluto signore della vita e degli avere de' popoli e con tutto ciò non è né può essere tiranno". Infatti, era obbligato "a non poter eleggere al grado di ministri altra specie di persone che quei manderini, i quali, perché sono ben

Mattia, in Dizionario Biografico degli Italiani, vol. XLI, Roma, Istituto dell'Enciclopedia Italiana, 1992.
121 Ivi, p. 237.
122 Ivi, p. 238.
123 Ivi, p. 239.
124 Ivi, p. 240. 
istruiti nella filosofia di Confucio, si nomano litterati”. Era dunque questa la peculiare condizione che impediva che i comportamenti dell'imperatore degenerassero in tirannia, perché "stando sempre in mezzo a uomini letterati e sapienti, non può, senza esporsi al pericolo del biasimo di tutto un popolo, ed anco a quello delle rivolte, far cose le quali alla giustizia ed alle altre virtù morali siano direttamente contrarie". ${ }^{125}$ Si determinava in tal modo quel che Doria veniva per davvero a considerare il "grande effetto, il quale a prima vista sembra paradosso, cioè che l'imperator della Cina essendo dispote non possa divenire tiranno" ${ }^{126}$ In realtà, il modello cinese non era che la riprova dell'assioma a partire dal quale Doria aveva elaborato il suo Politico alla moda contro "quella torta e perniciosa filosofia" 127 che era praticata dagli stati europei: infatti la "vera e retta filosofia è quella sola la quale, prescrivendo le norme della giustizia e delle altre virtù, fa argine alla tirannia. Ora fra i Cinesi la filosofia di Confucio, è quella la quale impedisce all'imperatore di poter divenir tiranno". ${ }^{128}$

Pronto a ricapitolare l'efficace applicazione di tale filosofia, ${ }^{129}$ Doria ne esaltava dichiaratamente la capacità di dar adito, nel comportamento dell'imperatore, a virtuose forme di omologazione:

$\mathrm{Al}$ certo questa legge è bastante a tenere entro i limiti della ragione qualunque principe, che fusse mal inclinato, e ciò perché è proprietà degli uomini questa seguente, cioè se un uomo reo ed ignorante è forzato a star sempre in mezzo a cento uomini savj e buoni, è forza che suo malgrado egli operi come saggio e come buono. ${ }^{130}$

Clausola assai forte di salvaguardia contro possibili degenerazioni

125 Ivi, p. 231.

126 Ivi, p. 232.

127 Ivi, p. 134.

128 Ivi, p. 232.

129 Ibidem: "Per lo più spesso fra i Cinesi l'Imperatore non è letterato, né filosofo esso stesso, ma fra i Cinesi questa è una salutare legge, cioè che l'Imperatore non possa innalzare alla dignità di ministro di alcun Magistrato altre persone che li manderini, i quali sono letterati, cioè filosofi divenuti sapienti nella filosofia di Confucio. Ora, in virtù di questa legge, l'Imperator della Cina è forzato a star sempre in mezzo a uomini sapienti, li quali con i loro saggi consigli fanno sempre argine e riparo alle passioni e alle false opinioni dell'Imperatore".

130 Ibidem. 
del comportamento del principe, tale legge poneva in grande evidenza il ruolo ricoperto dai mandarini nella stabilizzazione del sistema politico dell'impero:

Li manderini fanno nella Cina l'ufficio di tutori, e di mantenitori tutt'ad un tempo dell'autorità del Principe e della libertà del popolo; onde poi il popolo a cagione della loro sapienza, e dell'ufficio che fanno di tutori del popolo, non solo li considera ma li venera, onde poi se mai avviene che l'Imperatore voglia qualche azione fare, la quale sia troppo alle leggi dell'Imperio contraria, il popolo se ne disgusta, e l'Imperatore si pone in pericolo di una qualche rivoluzione contro di esso. ${ }^{131}$

Impegnati nella difficile arte di coniugare le opposte polarità che caratterizzavano l'assetto dell'impero, ovvero l'autorità del principe e la libertà del popolo, i mandarini proprio in questo modo venivano a implementare effettivamente "l'importante ufficio della politica". ${ }^{132}$ Per ciò stesso, dando vita a una ineludibile struttura burocratica, i mandarini costituivano, con la loro presenza, la pratica attenta della filosofia confuciana a unica condizione di distinzione sociale, di nobiltà: "In Cina v'è legge la quale ordina che solamente siano nobili li sapienti manderini, non v'è altro mezzo per ascendere alla nobiltà che quello della sapienza e della virtù". Questa considerazione portava la descrizione della "filosofia politica" che reggeva l'impero ad analizzare il percorso di selezione delle magistrature. Così, Doria non diversamente dai tanti autori che lo avevano preceduto, portava la propria attenzione ai "rigorosissimi esami" attraverso i quali avveniva la selezione dei candidati al mandarinato. Infatti

nella Cina v'è legge la quale ordina che niuno possa ascendere al grado di manderino, se non ha fatto fortissimi studj nella religione, nella morale, nella politica, nella legge; ed in conseguenza di ciò le leggi della Cina ordinano che per ascendere al grado di manderini li studiosi debbano passare tre volte un rigorosissimo esame, nel quale devono scrivere chiusi in una camera intorno agli quesiti che loro si danno, e ciò devono fare senza il soccorso di libri. ${ }^{133}$

131 Ivi, pp. 233-234

132 Ivi, p. 234.

133 Ibidem. 
Doria quindi concludeva la propria argomentazione affermando non solo che tali leggi erano "valevoli a fare che l'assoluta autorità dell'imperatore della Cina non possa in assoluta tirannide degenerare", ma che costituivano la ragione per cui l'impero si era conservato "per 4 mila anni grande e felice". ${ }^{134}$ Rappresentazione, questa, che incontrò però la netta opposizione di Montesquieu che, avendo svelato il segreto dell'esaltazione che l'impero aveva goduto soprattutto negli scritti dei missionari, ${ }^{135}$ intendeva per ciò stesso sortire dal mito e rimettere ordine tra le parole e le cose, affermando che in Cina

Si sono volute far regnare le leggi col Dispotismo. Ma ciò, che è congiunto col Dispotismo non ha più forza. Indarno questo Dispotismo urtato da' mali suoi ha tentato di incatenarsi: s'arma delle proprie catene, e diviene eziandio più terribile. È adunque la China uno Stato Dispotico, il cui principio è il timore. Può darsi, che nelle prime dinastie, non essendo l'Impero così esteso, il governo si dipartisse alquanto da questo spirito. Ma oggi la cosa è diversa. ${ }^{136}$

134 Ivi, p. 235

135 Ch. de Secondat barone di Montesquieu, Lo spirito delle leggi, cit., p. 231, ove, per spiegare l'eulogia del sistemo politico e sociale cinese confezionata e diffusa soprattutto dai gesuiti, Montesquieu sosteneva che: "Può darsi che i missionari siano stati ingannati da una apparenza d'ordine; ch'essi siano stati colpiti dall'esercizio perpetuo della volontà di un solo, dal quale essi stessi sono retti, e che si compiacciono di trovare alle corti dei re delle Indie. Inoltre, recandovisi per apportarvi grandi mutamenti, è per loro più facile convincere i principi che possono far tutto, che non persuadere i popoli che possono soffrire ogni cosa". Insomma, a leggere le loro opere con atteggiamento critico se ne svelava ben presto l'attitudine mistificatoria: "Dopo una serie di domande e di risposte sensate, il meraviglioso che se ne diceva è scomparso". Per un giudizio tutto affatto opposto, e assai più recente di quello proposto da Montesquieu, si vedano le importanti seppur rapide osservazioni sulla similitudine riscontrabile, quasi "compenetrazione reciproca", tra cultura dei gesuiti e quella dei mandarini offerte da A. Zingerle, La burocrazia cinese e il mutamento dinastico nel secolo XVII [...], cit., pp. 127-128.

136 Si cita dalla prima edizione italiana: Spirito delle Leggi del Signor di Montesquieu con le note dell'Abate Antonio Genovesi, Domenico Torres Negoziante di Libri, Napoli [colophon: Napoli, Presso Domenico Torres, 1777], vol. I, p. 264. 